Draft Version November 21, 2018

Preprint typeset using $\mathrm{LT}_{\mathrm{E}} \mathrm{X}$ style emulateapj v. 6/22/04

\title{
H I 21-CM ABSORPTION AND UNIFIED SCHEMES OF ACTIVE GALACTIC NUCLEI
}

\author{
S. J. Curran \\ School of Physics, University of New South Wales, Sydney NSW 2052, Australia \\ AND \\ M. T. Whiting \\ CSIRO Australia Telescope National Facility, PO Box 76, Epping NSW 1710, Australia \\ Draft version November 21, 2018
}

\begin{abstract}
In a recent study of $z \geq 0.1$ active galactic nuclei (AGN), we found that 21-cm absorption has never been detected in objects in which the ultra-violet luminosity exceeds $L_{\mathrm{UV}} \sim 10^{23} \mathrm{~W} \mathrm{~Hz}^{-1}$. In this paper, we further explore the implications that this has for the currently popular consensus that it is the orientation of the circumnuclear obscuring torus, invoked by unified schemes of AGN, which determines whether absorption is present along our sight-line. The fact that at $L_{\mathrm{UV}} \lesssim 10^{23}$ $\mathrm{W} \mathrm{Hz}{ }^{-1}$, both type-1 and type-2 objects exhibit a $50 \%$ probability of detection, suggests that this is not the case and that the bias against detection of $\mathrm{H} \mathrm{I}$ absorption in type- 1 objects is due purely to the inclusion of the $L_{\mathrm{UV}} \gtrsim 10^{23} \mathrm{~W} \mathrm{~Hz}^{-1}$ sources. Similarly, the ultra-violet luminosities can also explain why the presence of $21-\mathrm{cm}$ absorption shows a preference for radio galaxies over quasars and the higher detection rate in compact sources, such as CSS or GPS sources, may also be biased by the inclusion of high-luminosity sources. Being comprised of all 21-cm searched sources at $z \geq 0.1$, this is a necessarily heterogeneous sample, the constituents of which have been observed by various instruments. By this same token, however, the dependence on the UV luminosity may be an all encompassing effect, superseding the unified schemes model, although there is the possibility that the exclusive 21-cm non-detections at high UV luminosities could be caused by a bias towards gas-poor ellipticals. Additionally, the high UV fluxes could be sufficiently exciting/ionising the H I above 21 -cm detection thresholds, although the extent to which this is related to the neutral gas deficit in ellipticals is currently unclear.

Examining the moderate UV luminosity $\left(L_{\mathrm{UV}} \lesssim 10^{23} \mathrm{~W} \mathrm{~Hz}^{-1}\right.$ ) sample further, from the profile widths and offsets from the systemic velocities, we find no discernible differences between the two AGN types. This may suggest that the bulk of the absorption generally occurs in the galactic disk, which must therefore be randomly orientated with respect to the circumnuclear torus. Furthermore, we see no difference in the reddening between the two AGN types, indicating, like the 21-cm absorption, that the orientation of the torus has little bearing on this. We also find a correlation between $21-\mathrm{cm}$ line strength and the optical-near-infrared colour, which suggests that the reddening is caused by dust located in the large-scale, H I absorbing disk which intervenes the sight-line to the AGN.

Subject headings: galaxies: active - quasars: absorption lines - radio lines: galaxies - ultra violet: galaxies - galaxies: kinematic and dynamics - galaxies: high redshift
\end{abstract}

\section{INTRODUCTION}

Redshifted observations of the 21-cm spin-flip transition of neutral hydrogen $(\mathrm{H} \mathrm{I})$ trace the cool component of the gas in distant galaxies. Since the surface brightness has a $(1+z)^{4}$ dependence, the detection of the $21-\mathrm{cm}$ in emission is very difficult at redshifts of $z \geq 0.1$, and so the neutral gas in distant active galactic nuclei (AGN) is usually studied in absorption. Furthermore, since most published searches ${ }^{1}$ have been at redshifts of $z \lesssim 1$, there are generally no observations of the Lyman- $\alpha$ transition, which is redshifted into the optical bands at $z \geq 1.7$. Therefore, to date 21-cm absorption has been the most common probe of the neutral gas in the galaxies host to AGN, being detected in approximately $40 \%$ of $z \gtrsim 0.1$ cases (see Curran et al. 2008c).

In order to explain the detection rate, many studies invoke unified schemes of active galactic nuclei, which attempt to unify the many classes of luminous extra-

Electronic address: sjc@phys.unsw.edu.au

1 Prior to the $z \sim 3$ survey of Curran et al. (2008c). galactic object, a key element of which is the presence of a torus of highly obscuring circumnuclear material: In these schemes, the appearance of the object is dependent upon the orientation of this material along our line-of-sight to the nucleus (Osterbrock 1978; Antonucci \& Miller 1985; Miller \& Goodrich 1987; Antonucci 1993; Urry \& Padovani 1995), with the popular consensus being that only type- 2 objects present a dense column of intervening gas, which can absorb in 21-cm (see Jaffe \& McNamara 1994; Conway \& Blanco 1995, figure 2 of Curran et al. 2008b). Other observational examples of this include:

1. From a survey for $21-\mathrm{cm}$ absorption in 23 radio galaxies, Morganti et al. (2001) find that of the five detections, four occur in sources which could be considered type- 2 objects, whereas there is only one detected case for a type-1 object.

2. From a study of 49 gigahertz peaked spectrum (GPS) and compact steep spectrum (CSS) sources, Pihlström et al. (2003) find that 21-cm absorption 
is more likely to arise in objects classified as galaxies, rather than in quasars. Since the former are generally considered to be type- 2 objects, while latter are type- 1 objects, this is consistent with the orientation of the central obscuration playing a major rôle in producing strong 21-cm absorption along our sight-line.

3. Also, from a study of 27 GPSs and CSSs, Gupta et al. (2006) find that 21-cm absorption is twice as likely to be detected in the galaxies than in the quasars of the sample, again suggesting that the absorption occurs in the dense sub-parsec torus.

4. From a sample of 23 galaxies and 9 quasars, Gupta \& Saikia (2006b) find that 15 of the galaxies exhibit 21-cm absorption, compared to just a single case for the quasars. Like Pihlström et al. (2003), this is consistent with unified schemes, where galaxies are host to edge-on obscurations, whereas quasars have their tori oriented more faceon.

However, the situation may be more complex than this with evidence that 21-cm absorption may also be due to in-falling gas or outflows (e.g. Vermeulen et al. 2003; Morganti et al. 2005b, 2007), and if these are directed along the radio jet axis, we would expect outflows of neutral gas to render absorption detectable towards type-1 sources. Whether due to an outflow or the presence of an intervening circumnuclear obscuration, these scenarios are consistent with unified schemes of AGN, playing a major rôle in whether $\mathrm{H}$ I 21-cm absorption is detected.

Therefore, from these possibilities in addition to absorption by the large reservoir of neutral gas in the galactic disk, we may expect a high $21-\mathrm{cm}$ detection rate in distant radio galaxies and quasars. However, from a recent survey of the host galaxies of $z \geq 2.9$ quasars, Curran et al. (2008c) found no evidence of absorption in any of the ten sources searched. Upon an analysis of the spectral types of the targets, as well as those of all the other $z \geq 0.1$ published searches, they found the nondetections all to be type- 1 objects, as are many of the lower redshift non-detections (see Fig. 1). Superficially, this suggests that the orientation of the circumnuclear obscuration may be key in the detection of 21-cm absorption, although there may also be other effects at play, the evidence for which we discuss in this paper.

\section{FACTORS AFFECTING THE 21-CM DETECTION RATE}

\subsection{Luminosities}

\subsubsection{Ultra-violet luminosity}

As stated above, at redshifts of $z \gtrsim 3$ Curran et al. (2008c) detected no 21-cm absorption down to sensitivities sufficient for most of the current detections, $N_{\mathrm{HI}} \lesssim$ $10^{18} \cdot\left(T_{\mathrm{s}} / f\right) \mathrm{cm}^{-2}$ per channel (Tables $11 \&$ 2, where we include the $z \geq 0.1$ searches which were previously missed $\left.^{2}\right)$. From an analysis of the optical photometry, the target absorption systems of Curran et al. (2008c)

2 We have added the $z \geq 0.1$ detections $1549-79 \& 3 \mathrm{C} 433$ and the $z>0.1$ non-detections 0035-024, 0723-008, 1356+022 $\& 1615+02 \overline{8}$, where the photometry and AGN type have been obtained/determined from Allen et al. (1982); Wall \& Peacock (1985); Lipovetsky et al. (1988); Smith \& Heckman (1989); are found to be located in quasars with high ultra-violet $(\lambda \approx 1216 \AA)$ luminosities $\left(L_{\mathrm{UV}} \gtrsim 10^{23} \mathrm{~W} \mathrm{~Hz}^{-1}, \mathrm{Fig}[1)^{3}\right.$, which suggests that the gas may have a significant ionisation fraction in all of these sources. In light of this, the large non-detection rate at high redshift is perhaps expected due to the flux limited nature of optical surveys, which selects only the most UV bright objects at these distances. Note, however, that this effect is also apparent for previously searched lower redshift $(z \lesssim 1)$ sources, a trait which was previously unknown.

In order to determine the significance of this UV segregation, in Table 3 we summarise the binomial probabilities of the observed distributions occuring by chance, given that a 21-cm detection and non-detection are equally probable at a given UV luminosity. From this, we see that below each UV luminosity cut-off there is no bias, with the likelihood of a detection staying close to $50 \%$. On the other hand, above the cut-off the probabilities of the observed distributions resulting from an unbiased sample are small, dropping dramatically at $L_{\mathrm{UV}}>10^{23} \mathrm{~W} \mathrm{~Hz}{ }^{-1}$. Since all luminosities above the cut-off are included, the most luminous sources could well dominate the upper partitions in Table 3. which does appear to be evident from the vertical histogram of Fig. 1. As is also illustrated by the histogram, above $L_{\mathrm{UV}} \gtrsim 10^{20} \mathrm{~W} \mathrm{~Hz}^{-1}$ the number of non-detections outweighs the number of detections in each bin, which may suggest that at all values the ultra-violet luminosity introduces a bias against 21-cm absorption. However, this could also be explained by other effects which could make non-detections more numerous, e.g. a larger proportion of type- 1 objects (presuming that unified schemes were key in determining whether 21-cm absorption could be detected, see below). What is clear, however, is that $21-\mathrm{cm}$ has yet to be detected at $L_{\mathrm{UV}}>10^{23} \mathrm{~W} \mathrm{~Hz}^{-1}$ and that the probability of this distribution occuring by chance is very small (Table 3).

A quantitative estimate of the critical UV luminosity may be obtained by examining the detection proportions above and below certain values of $L_{\mathrm{UV}}$. We define the statistic

$$
T=\frac{\hat{p}_{1}-\hat{p}_{2}}{\sqrt{\hat{p}(1-\hat{p})\left(N_{1}^{-1}+N_{2}^{-1}\right)}},
$$

where $\hat{p}_{1}=X_{1} / N_{1}$ and $\hat{p}_{2}=X_{2} / N_{2}$ are the two measured proportions and $\hat{p}=\left(X_{1}+X_{2}\right) /\left(N_{1}+N_{2}\right)$ is the total proportion. This has the standard normal distribution under the null hypothesis that the proportions $\left(\hat{p}_{1}\right.$ and $\hat{p}_{2}$ ) are the same, i.e. that the UV luminosity does not affect the $21-\mathrm{cm}$ detection rate. Testing the statistic in steps of $\Delta \log _{10} L_{\mathrm{UV}}=0.1$, we reject the null hypothesis for all UV luminosities of $\log _{10} L_{\mathrm{UV}} \geq 22.5$, where the difference between the two proportions is significant at $\geq 3 \sigma$ (Fig. 2] left). Additionally, as found above, the sources below the $L_{\text {UV }}$ cut-off always exhibit a $\approx 50 \%$ detection rate (Table 3), which is the natural rate in the absence of high UV luminosities.

\subsubsection{Radio luminosity}

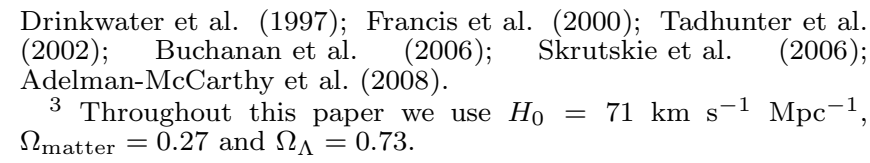

Drinkwater et al. (1997); Francis et al. (2000); Tadhunter et al. Adelman-McCarthy et al. (2008).

$\Omega_{\text {matter }}=0.27$ and $\Omega_{\Lambda}=0.73$. 
TABLE 1

The $z \geq 0.1$ SOURCES DETECTED IN 21-CM ABSORPTION.

\begin{tabular}{|c|c|c|c|c|c|c|c|c|c|c|c|c|}
\hline \multirow[t]{2}{*}{ Source } & \multirow[t]{2}{*}{ Class } & \multirow[t]{2}{*}{$z_{\mathrm{em}}$} & \multirow{2}{*}{$\begin{array}{c}B \\
{[\mathrm{mag}]}\end{array}$} & \multirow{2}{*}{$\begin{array}{c}V \\
{[\mathrm{mag}]}\end{array}$} & \multirow{2}{*}{$\left.\begin{array}{c}R \\
{[\mathrm{mag}]}\end{array}\right]$} & \multirow{2}{*}{$\begin{array}{c}K \\
{[\mathrm{mag}]}\end{array}$} & \multirow{2}{*}{$\begin{array}{r}\log L_{\mathrm{UV}} \\
{\left[\mathrm{W} \mathrm{Hz}{ }^{-1}\right]} \\
\end{array}$} & \multirow[t]{2}{*}{ Type } & \multirow{2}{*}{$\begin{array}{c}\log _{10} N_{\mathrm{HI}} \\
\left(f / T_{\mathrm{s}}\right)\left[\mathrm{cm}^{-2} / \mathrm{K}\right]\end{array}$} & \multirow[t]{2}{*}{ ID } & \multicolumn{2}{|r|}{ References } \\
\hline & & & & & & & & & & & Spe. & Con. \\
\hline J0025-2602 & Gal & 0.3220 & 20.300 & - & 18.084 & 15.674 & 20.100 & 2 & 18.36 & CSS & V03 & T02 \\
\hline $0108+388$ & Gal & 0.6685 & - & - & 22.000 & 16.690 & 20.309 & 2 & 19.90 & GPS & C98 & P88,B90,O98,Z02 \\
\hline $\mathrm{J} 0141+1353$ & Gal & 0.6210 & 22.327 & 20.920 & 20.876 & 16.680 & 20.777 & 2 & 18.04 & CSS & V03 & F89,S95 \\
\hline $\mathrm{J} 0410+7656$ & Gal & 0.5985 & - & - & 21.200 & - & - & 2 & 18.40 & GPS & V03 & D95,S95a \\
\hline $\mathrm{J} 0414+0534$ & Gal & 2.6365 & 24.100 & 23.800 & 21.270 & 13.540 & 22.188 & 1 & 18.88 & - & M99 & - \\
\hline $\mathrm{J} 0431+2037$ & Gal & 0.2190 & 22.174 & - & 19.085 & 14.924 & 18.039 & - & 18.54 & GPS & V03 & D95,S01a \\
\hline $0500+019$ & Gal & 0.5846 & 22.500 & 21.350 & 20.682 & 15.430 & 20.367 & 2 & 18.79 & FSRS & C98 & S01b \\
\hline 3C 190 & QSO & 1.1946 & 19.976 & 17.460 & 18.972 & 15.300 & 22.825 & 1 & 19.6 & CSS & I03 & - \\
\hline J0834+5534 & Gal & 0.2420 & 18.921 & 17.390 & 17.180 & 14.180 & 20.719 & 1 & 18.03 & $\mathrm{RG}$ & V03 & W85 \\
\hline J0901+2901 & Gal & 0.1940 & 19.321 & 18.078 & 18.600 & 15.200 & 21.280 & 1 & 17.04 & CSS & V03 & A95 \\
\hline $0902+343$ & Gal & 3.3980 & - & 23.800 & 23.500 & 19.900 & 22.422 & - & 18.49 & - & U91 & - \\
\hline J0909+4253 & QSO & 0.6700 & 18.960 & 19.049 & 18.220 & 14.860 & 22.699 & 2 & 18.09 & CSS & V03 & V92 \\
\hline $\mathrm{J} 1124+1919$ & Gal & 0.1650 & 22.082 & 21.448 & 20.513 & 15.930 & 19.190 & - & 18.70 & CSS & G06 & S90,S95b \\
\hline $12032+1707$ & Gal & 0.2170 & 18.758 & - & 17.327 & 14.864 & 20.949 & 2 & 18.74 & OHM & P05 & - \\
\hline $\mathrm{J} 1206+6413$ & Gal & 0.3710 & 21.847 & 20.790 & 19.910 & - & 19.908 & 1 & 18.29 & CSS & V03 & S95a,L98 \\
\hline $\mathrm{J} 1326+3154$ & Gal & 0.3700 & 21.367 & 19.822 & 18.882 & 14.940 & 19.638 & 2 & 17.85 & GPS & V03 & M81,F96 \\
\hline $4 \mathrm{C} 12.50$ & QSO & 0.1217 & 16.615 & 16.050 & 15.718 & 13.216 & 21.736 & 2 & 18.79 & GPS & M89 & L03 \\
\hline $\mathrm{J} 1357+4354$ & Gal & 0.6460 & - & 22.708 & 20.951 & - & 18.620 & - & 19.52 & GPS & V03 & Т96 \\
\hline $\mathrm{J} 1400+6210$ & Gal & 0.4310 & 22.137 & 20.373 & 19.530 & 16.130 & 19.459 & 2 & 18.27 & GPS & V03 & D95 \\
\hline $1413+135$ & QSO & 0.2467 & 21.055 & 20.000 & 18.461 & 14.928 & 19.105 & 1 & 19.11 & CSS & C92 & P96,P00 \\
\hline $1504+377$ & Gal & 0.6715 & - & 21.808 & 20.800 & 16.100 & 20.295 & 2 & 19.65 & FSRS & C98 & VLBA \\
\hline 1549-79 & Gal & 0.1501 & - & 18.800 & - & 12.407 & 19.965 & 1 & 18.56 & CFS & M01 & same \\
\hline $\mathrm{J} 1815+6127$ & QSO & 0.6010 & 21.272 & - & 19.122 & - & 20.665 & 1 & 18.64 & GPS & V03 & T94 \\
\hline $\mathrm{J} 1816+3457$ & Gal & 0.2448 & 20.342 & 一 & 18.459 & 15.525 & 20.034 & - & 18.71 & GPS & P00 & same \\
\hline $\mathrm{J} 1821+3942$ & Gal & 0.7980 & 19.598 & 一 & 18.135 & 15.023 & 22.202 & 1 & 18.22 & CSS & V03 & D95,S01a \\
\hline $\mathrm{J} 1944+5448$ & Gal & 0.2630 & 21.732 & - & 18.591 & 15.000 & 18.424 & 2 & 18.69 & GPS & V03 & S01a,X95 \\
\hline $\mathrm{J} 1945+7055$ & Gal & 0.1010 & 18.726 & - & 17.199 & 13.369 & 20.067 & 2 & 18.5 & GPS & P99 & Т97 \\
\hline $\mathrm{J} 2052+3635$ & Gal & 0.3550 & 22.083 & - & 21.200 & - & 20.648 & 1 & 18.86 & GPS & V03 & P81 \\
\hline 3C 433 & Gal & 0.1016 & 17.660 & 16.350 & - & 12.891 & 21.739 & 2 & 18.36 & $\mathrm{RG}$ & M89 & - \\
\hline $\mathrm{J} 2255+1313$ & QSO & 0.5430 & 19.535 & 19.590 & 19.190 & - & 22.530 & 2 & 17.62 & CSS & V03 & A95 \\
\hline $\mathrm{J} 2316+0405$ & Gal & 0.2199 & 18.595 & 17.440 & 17.220 & 13.991 & 21.081 & 2 & 17.85 & BLRG & V03 & T03 \\
\hline $\mathrm{J} 2355+4950$ & Gal & 0.2379 & 21.101 & - & 18.400 & 15.112 & 18.940 & 2 & 18.45 & GPS & V03 & P95,T00 \\
\hline
\end{tabular}

Note. - references in the penultimate column $(3 \mathrm{C} 190=0758+143,4 \mathrm{C} 12.50=1345+12,3 \mathrm{C} 433=2121+24,3 \mathrm{C} 452=$ J2245+3941). The references for the magnitudes and AGN types are given in Curran et al. (2008c) [see also footnote 2]. The $1216 \AA$ A luminosities and AGN types are calculated and determined as described in Curran et al. (2008c) and the final four columns give the H I column density, radio ID (see notes) and the 21-cm search (spe.) \& high resolution radio imaging (where available, con.) references.

BLRG - broad line radio galaxy, CFS - compact flat spectrum, CSO - compact symmetric object, CSS - compact steep spectrum source, EORG - end-on radio galaxy, EORQ - end-on radio, quasar, FRI - Fanaroff Riley type-1/BL Lac object, FRII - Fanaroff Riley type-2, FSRQ - flat-spectrum radio quasar, FSRQ - flat spectrum radio source, FSSO - flat-spectrum symmetric object, HFP - high frequency peaker galaxy, NLRG - narrow-line radio galaxy, OHM-OH megamaser, RG - radio galaxy, RQ - radio quasar.

References. - Spectral: D85 - de Waard et al. (1985), M89 - Mirabel (1989), V89 - van Gorkom et al. (1989), U91 Uson et al. (1991), C92 - Carilli et al. (1992), C98 - Carilli et al. (1998), M99 - Moore et al. (1999), P99 - Peck et al. (1999), P00 - Peck et al. (2000), M01 - Morganti et al. (2001), I03 - Ishwara-Chandra et al. (2003), P03 - Pihlström et al. (2003), V03 - Vermeulen et al. (2003), P05 - Pihlström et al. (2005), C06 - Curran et al. (2006), G06 - Gupta et al. (2006), GS06 - Gupta \& Saikia (2006a), O06 - Orienti et al. (2006), C07 - Carilli et al. (2007), C08 - Curran et al. (2008c). Continuum: J77 - Jenkins et al. (1977), M81 - Mutel et al. (1981), P81 - Phillips \& Mutel (1981), B82 - Browne et al. (1982) U83 Ulvestad et al. (1983), A85 - Antonucci \& Ulvestad (1985), W85 - Whyborn et al. (1985), G88 - Giovannini et al. (1988), P88 - Pearson \& Readhead (1988), F89 - Fanti et al. (1989), S89a - Spencer et al. (1989), S89b - Stanghellini et al. (1999), B90 Baum et al. (1990), S90 - Strom et al. (1990), A91 - Akuior et al. (1991), V92 - van Breugel et al. (1992), M93 - Murphy et al. (1993), T94 - (Tavlor et al. 1994), A95 - Akuior \& Garrington (1995), D95 - Dallacasa et al. (1995), N95 - Neff et al. (1995), P95 - Polatidis et al. (1995), S95a - Saikia et al. (1995), S95b - Sanghera et al. (1995), X95 - Xu et al. (1995), B96-Bondi et al. (1996), F96 - Fev et al. (1996), P96 - Perlman et al. (1996), T96 - Tavlor et al. (1996a), T97 - Tavlor \& Vermeulen (1997), B98 - Bondi et al. (1998), L98 - Lüdke et al. (1998), S98 - Saikia \& Kulkarni (1998), O98 - Owsianik et al. (1998), R99 - Reid et al. (1999), F00 - Fomalont et al. (2000), T00 - Tavlor et al. (2000), S01a - Saikia et al. (2001), S01b - Stanghellini et al. (2001), B02 - Beaslev et al. (2002), T02 - Tzioumis et al. (2002), Z02 - Zensus et al. (2002), F03 - Fomalont et al. (2003), L03 Lister et al. (2003), T03 - Thomasson et al. (2003), P05 - Petrov et al. (2005), K09 - Kanekar et al. (2009), VLBA - VLBA calibrator. 


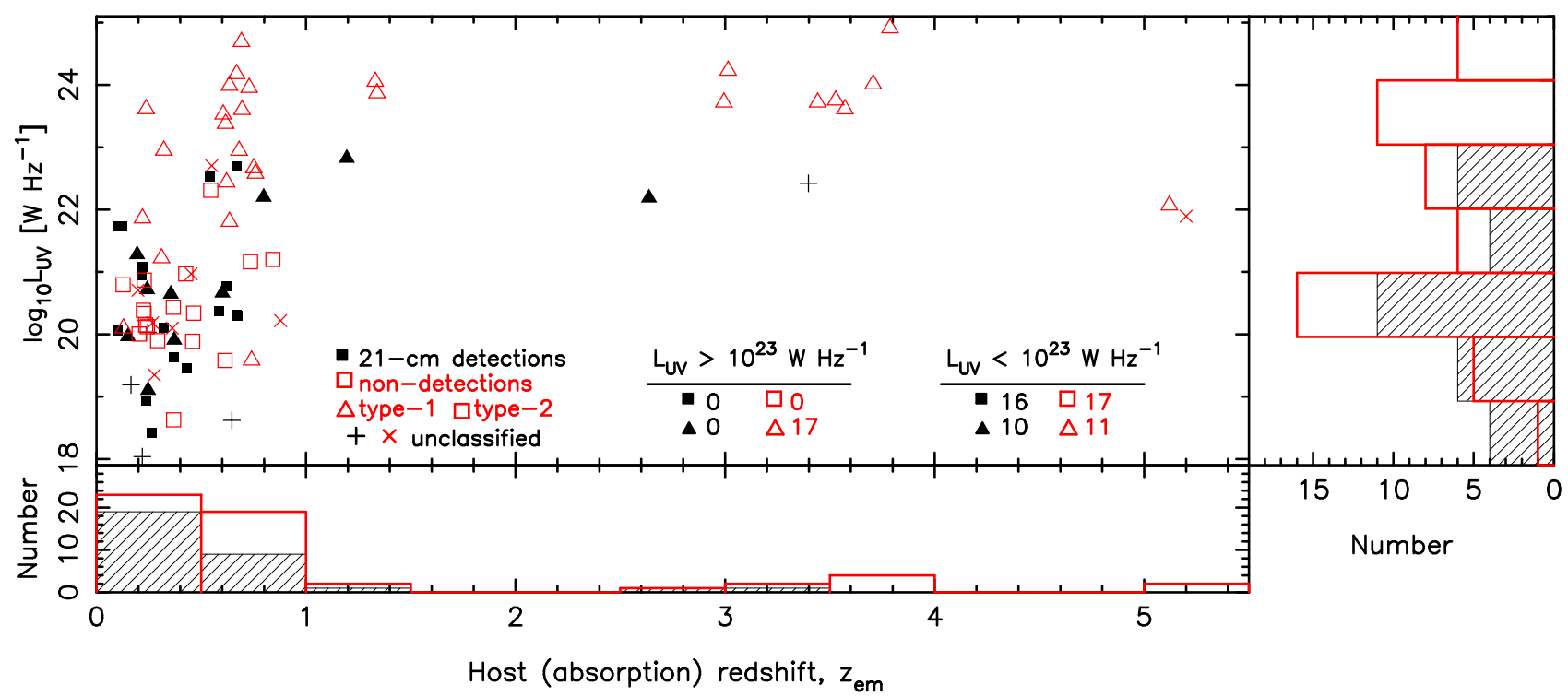

FIG. 1. - The ultra-violet luminosity-redshift distribution for the $z \geq 0.1$ radio galaxies and quasars searched in associated 21-cm absorption. The filled symbols/hatched histogram represent the 21-cm detections and the unfilled symbols/unfilled histogram the nondetections. The shapes represent the AGN classifications, with triangles representing type- 1 objects and squares type-2s $(+$ and $\times$ designate an undetermined AGN type for a detection and non-detection, respectively). The legend shows the number of each AGN type according to the $L_{\mathrm{UV}}=10^{23} \mathrm{~W} \mathrm{~Hz}^{-1}$ partition. Updated from (Curran et al. 2008c).
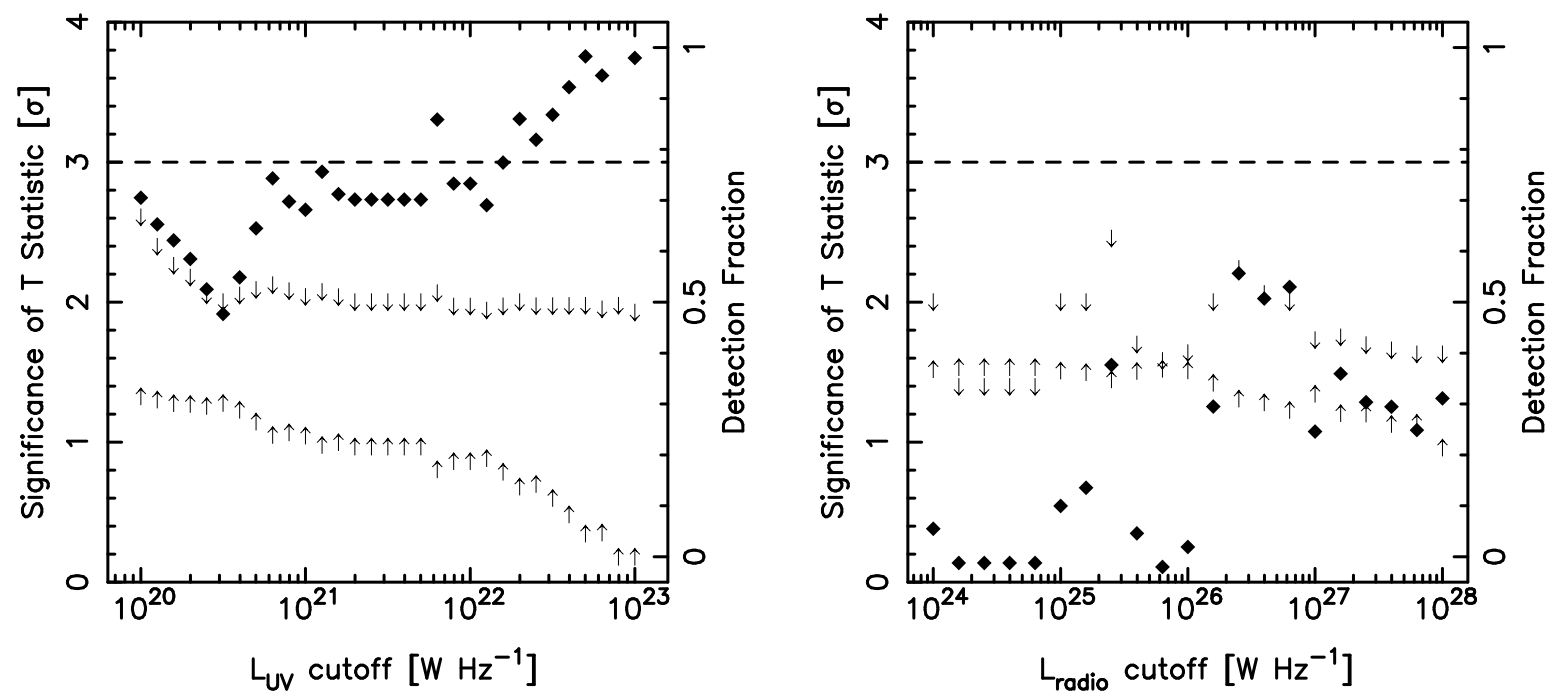

FIG. 2.- The significance of the T-statistic of the difference in the proportions, $\hat{p}_{1}$ and $\hat{p}_{2}$, for various critical luminosities. The left panel shows this for the ultra-violet and the right panel for the rest-frame $1420 \mathrm{MHz}$ continuum luminosity. The ordinate label on the right-hand side of each panel shows the detection fraction above (upward arrows) and below (downward) the cut-off. 
TABLE 2

As Table 1 but for the non-detections, where the H i COlumn Density limits are Quoted at a $3 \sigma$ Level Per Channel.

\begin{tabular}{|c|c|c|c|c|c|c|c|c|c|c|c|c|}
\hline \multirow[t]{2}{*}{ Source } & \multirow[t]{2}{*}{ Class } & \multirow[t]{2}{*}{$z_{\mathrm{em}}$} & \multirow{2}{*}{$\begin{array}{c}B \\
{[\mathrm{mag}]}\end{array}$} & \multirow{2}{*}{$\begin{array}{c}V \\
{[\mathrm{mag}]}\end{array}$} & \multirow{2}{*}{$\begin{array}{c}R \\
{[\mathrm{mag}]}\end{array}$} & \multirow{2}{*}{$\begin{array}{c}K \\
{[\mathrm{mag}]}\end{array}$} & \multirow{2}{*}{$\begin{array}{r}\log L_{\mathrm{UV}} \\
{\left[\mathrm{W} \mathrm{Hz}^{-1}\right]}\end{array}$} & \multirow{2}{*}{ Type } & \multirow{2}{*}{$\begin{array}{c}\log _{10} N_{\mathrm{HI}} \\
\left(f / T_{\mathrm{s}}\right)\left[\mathrm{cm}^{-2} / \mathrm{K}\right]\end{array}$} & \multirow[t]{2}{*}{ ID } & \multicolumn{2}{|c|}{ References } \\
\hline & & & & & & & & & & & Spe. & Con. \\
\hline $\mathrm{J} 0003+2129$ & QSO & 0.4520 & 21.005 & 20.580 & 19.650 & - & 20.971 & - & $<19.61$ & $\mathrm{HFP}$ & O06 & VLBA \\
\hline 0035-024 & Gal & 0.2197 & 19.110 & 17.920 & - & 14.494 & 21.862 & 1 & $<17.67$ & FRII & M01 & VLBA \\
\hline $0131-001$ & QSO & 0.8790 & 23.340 & 22.500 & 20.780 & 16.780 & 20.221 & - & $<18.28$ & - & $\mathrm{C} 06$ & VLBA \\
\hline J0157-1043 & QSO & 0.6160 & 17.504 & - & 17.039 & - & 23.380 & 1 & $<17.98$ & EORQ & V03 & R99 \\
\hline J0201-1132 & QSO & 0.6690 & 16.232 & - & 16.073 & 13.860 & 24.176 & 1 & $<17.80$ & EORQ & V03 & R99 \\
\hline $\mathrm{J} 0224+2750$ & Gal & 0.3102 & 19.502 & - & 18.263 & 15.250 & 21.225 & 1 & $<18.14$ & CSS & V03 & S95a,b \\
\hline 0335-122 & QSO & 3.4420 & 21.018 & 20.110 & 20.199 & 17.510 & 23.722 & 1 & $<18.32$ & - & $\mathrm{C} 08$ & VLBA \\
\hline $0347-211$ & QSO & 2.9940 & 20.476 & - & 20.297 & 17.900 & 23.722 & 1 & $<18.38$ & - & $\mathrm{C} 08$ & VLBA \\
\hline $\mathrm{J} 0348+3353$ & Gal & 0.2430 & 20.723 & - & 19.110 & 14.390 & 20.121 & 2 & $<18.12$ & CSS & V03 & D95 \\
\hline $\mathrm{J} 0401+0036$ & Gal & 0.4260 & 20.200 & 19.010 & 18.532 & - & 20.969 & 2 & $<18.03$ & EORG & V03 & N95 \\
\hline $\mathrm{J} 0521+1638$ & QSO & 0.7590 & 19.370 & 18.840 & 18.480 & 15.380 & 22.580 & 1 & $<17.65$ & CSS & V03 & F89,A91,S95a \\
\hline 0537-286 & QSO & 3.0140 & 19.290 & - & 18.789 & 16.770 & 24.231 & 1 & $<18.45$ & FSRQ & $\mathrm{C} 08$ & K09 \\
\hline J0542+4951 & QSO & 0.5450 & 18.450 & 17.800 & 17.210 & - & 22.311 & 2 & $<17.45$ & CSS & V03 & L98 \\
\hline J0556-0241 & Gal & 0.2350 & 20.968 & - & 19.533 & 一 & 20.150 & 2 & $<18.77$ & GPS & V03 & $\mathrm{P} 05$ \\
\hline $\mathrm{J} 0609+4804$ & Gal & 0.2769 & 21.198 & - & 18.767 & 一 & 19.349 & - & $<17.79$ & EORG & V03 & N95 \\
\hline $\mathrm{J} 0709+7449$ & Gal & 0.2921 & 19.982 & - & 17.540 & 13.790 & 19.898 & 2 & $<18.30$ & FRII & V03 & - \\
\hline 0723-008 & QSO & 0.1273 & 17.39 & 16.57 & 15.82 & 13.166 & 20.793 & 2 & $<17.76$ & CFS & V89 & B96 \\
\hline $\mathrm{J} 0741+3112$ & QSO & 0.6350 & 16.517 & 16.100 & 16.322 & 16.100 & 23.990 & 1 & $<17.97$ & GPS & V03 & S98,S01 \\
\hline J0815-0308 & Gal & 0.1980 & 18.490 & 16.940 & 16.797 & 13.858 & 20.707 & - & $<18.16$ & EORG & V03 & N95 \\
\hline $\mathrm{J} 0840+1312$ & QSO & 0.6808 & 18.370 & 17.940 & 17.622 & 15.280 & 22.947 & 1 & $<17.69$ & RQ & V03 & VLBA \\
\hline J0913+5919 & QSO & 5.1200 & - & 23.281 & 24.948 & - & 22.071 & 1 & $<19.34$ & $\mathrm{CSO}$ & $\mathrm{C} 07$ & - \\
\hline J0924-2201 & Gal & 5.2000 & - & - & 25.850 & - & 21.893 & - & $<18.34$ & $\mathrm{CSO}$ & $\mathrm{C} 07$ & - \\
\hline J0927+3902 & QSO & 0.6948 & 17.064 & - & 16.486 & 一 & 23.603 & 1 & $<17.97$ & EORG & V03 & B82 \\
\hline $\mathrm{J} 0939+8315$ & Gal & 0.6850 & - & - & 20.140 & - & - & 2 & $<17.67$ & EORG & V03 & $\mathrm{J} 77$ \\
\hline J0943-0819 & Gal & 0.2280 & 19.401 & - & 18.100 & 14.750 & 20.868 & 2 & $<18.08$ & GPS & V03 & B02 \\
\hline J0954+7435 & Gal & 0.6950 & - & - & 21.700 & - & - & - & $<18.37$ & $\mathrm{RG}$ & V03 & F00 \\
\hline $\mathrm{J} 1035+5628$ & Gal & 0.4590 & - & 21.244 & 20.200 & - & 19.889 & 2 & $<18.12$ & GPS & V03 & T94,P00 \\
\hline $\mathrm{J} 1120+1420$ & Gal & 0.3620 & - & 20.935 & 20.100 & 17.100 & 20.098 & - & $<17.76$ & GPS & V03 & B98 \\
\hline $\mathrm{J} 1159+2914$ & QSO & 0.7290 & 17.489 & 18.113 & 17.652 & - & 23.955 & 1 & $<18.26$ & EORG & V03 & A 85 \\
\hline 1228-113 & QSO & 3.5280 & 22.010 & - & 19.115 & 16.370 & 23.754 & 1 & $<18.63$ & - & $\mathrm{C} 08$ & VLBA \\
\hline $\mathrm{J} 1252+5634$ & QSO & 0.3210 & 17.760 & 17.930 & 17.660 & - & 22.949 & 1 & $<17.83$ & CSS & V03 & S95a,L98 \\
\hline J1308-0950 & Gal & 0.4640 & 20.767 & 20.500 & 18.439 & - & 20.340 & 2 & $<18.11$ & CSS & V03 & T02 \\
\hline $\mathrm{J} 1313+5458$ & QSO & 0.6130 & - & 21.735 & 20.374 & - & 19.581 & 2 & $<18.23$ & $\mathrm{RQ}$ & V03 & Т94 \\
\hline 1351-018 & QSO & 3.7070 & 21.030 & 19.696 & 19.277 & 17.070 & 24.014 & 1 & $<18.41$ & - & $\mathrm{C} 08$ & - \\
\hline $1356+022$ & QDO & 1.330 & - & 17.436 & - & 14.537 & 24.055 & 1 & $<17.88$ & FSSO & D85 & VLBA \\
\hline $\mathrm{J} 1421+4144$ & Gal & 0.3670 & 20.496 & 19.330 & 18.560 & 15.910 & 20.435 & 2 & $<17.82$ & CSS & V03 & A95 \\
\hline $\mathrm{J} 1443+7707$ & Gal & 0.2670 & - & - & 18.730 & - & - & 2 & $<18.15$ & CSS & V03 & L98 \\
\hline $1450-338$ & Gal & 0.3680 & 22.520 & 20.400 & 19.390 & 15.230 & 18.629 & 2 & $<17.83$ & - & $\mathrm{C} 06$ & VLBA \\
\hline $1535+004$ & QSO & 3.4970 & - & - & - & 19.540 & - & - & $<18.22$ & FSSO & $\mathrm{C} 06$ & VLBA \\
\hline $\mathrm{J} 1540+1447$ & QSO & 0.6050 & 17.480 & 17.000 & 17.240 & 13.640 & 23.529 & 1 & $<17.77$ & EORG & V03 & U83 \\
\hline $\mathrm{J} 1546+0026$ & Gal & 0.5500 & 19.730 & 18.900 & - & 16.420 & 22.703 & - & $<18.00$ & GPS & V03 & $\mathrm{P} 00$ \\
\hline $1615+028$ & QSO & 1.339 & 18.010 & 17.750 & 17.310 & 15.890 & 23.869 & 1 & $<18.30$ & FSSO & D85 & - \\
\hline $\mathrm{J} 1623+6624$ & Gal & 0.2030 & 19.477 & - & 17.430 & - & 20.004 & 2 & $<18.48$ & HFP & O06 & - \\
\hline $\mathrm{J} 1642+6856$ & QSO & 0.7510 & 19.723 & - & 19.219 & - & 22.667 & 1 & $<18.10$ & EORG & V03 & M93 \\
\hline $\mathrm{J} 1658+0741$ & QSO & 0.6210 & 19.993 & - & 19.598 & - & 22.441 & 1 & $<18.17$ & EORG & V03 & M93 \\
\hline $\mathrm{J} 1823+7938$ & Gal & 0.2240 & 19.269 & - & 17.415 & 13.866 & 20.385 & 2 & $<19.44$ & GPS & V03 & T94 \\
\hline $\mathrm{J} 1829+4844$ & QSO & 0.6920 & 16.260 & - & 16.860 & 14.250 & 24.692 & 1 & $<17.26$ & CSS & V03 & L98 \\
\hline $\mathrm{J} 1831+2907$ & Gal & 0.8420 & 21.917 & - & 20.200 & - & 21.201 & 2 & $<18.21$ & CSS & V03 & S89a \\
\hline $\mathrm{J} 1845+3541$ & Gal & 0.7640 & - & - & 21.900 & - & - & 2 & $<19.02$ & GPS & V03 & X95 \\
\hline 1937-101 & QSO & 3.7870 & 18.800 & - & 17.188 & 13.816 & 24.910 & 1 & $<18.11$ & - & $\mathrm{C} 08$ & VLBA \\
\hline $\mathrm{J} 2022+6136$ & Gal & 0.2270 & 19.830 & - & 18.146 & - & 20.334 & 2 & $<17.56$ & GPS & V03 & F00 \\
\hline J2137-2042 & Gal & 0.6350 & 20.400 & - & 19.286 & - & 21.808 & 1 & $<18.05$ & CSS & V03 & T02,F03 \\
\hline $2149+056$ & QSO & 0.7400 & 23.700 & 22.050 & 20.850 & 17.170 & 19.582 & 1 & $<18.38$ & FSRS & C98 & S89b \\
\hline $2215+02$ & QSO & 3.5720 & 21.840 & 20.420 & 20.140 & 19.340 & 23.613 & 1 & $<17.57$ & FSSO & $\mathrm{C} 08$ & VLBA \\
\hline $\mathrm{J} 2250+1419$ & QSO & 0.2370 & 16.760 & 16.640 & 17.243 & - & 23.616 & 1 & $<18.09$ & CSS & V03 & - \\
\hline 2300-189 & Gal & 0.1290 & 18.430 & - & 16.569 & 13.060 & 20.099 & 1 & $<18.20$ & - & $\mathrm{C} 06$ & VLBA \\
\hline $\mathrm{J} 2321+2346$ & Gal & 0.2680 & 20.315 & - & 18.468 & 14.710 & 20.187 & - & $<18.28$ & EORG & V03 & G88 \\
\hline $\mathrm{J} 2344+8226$ & QSO & 0.7350 & 21.769 & - & 20.220 & 15.850 & 21.165 & 2 & $<17.85$ & GPS & V03 & D95,S01b \\
\hline
\end{tabular}


TABLE 3

The inCIDENCE OF DETECTIONS For VARIOUS UV LUMinOSITY PARTITIONS.

\begin{tabular}{lcccccccc}
\hline$L_{\mathrm{UV}}$ & \multicolumn{3}{c}{ For luminosities $<L_{\mathrm{UV}}$} & \multicolumn{5}{c}{ For luminosities $>L_{\mathrm{UV}}$} \\
$\mathrm{W} \mathrm{Hz}^{-1}$ & $k / n$ & rate & $P(\leq k / n)$ & $S(\leq k / n)$ & $k / n$ & rate & $P(\leq k / n)$ & $S(\leq k / n)$ \\
\hline $10^{20}$ & $10 / 17$ & $59 \%$ & 0.31 & $1.00 \sigma$ & $21 / 68$ & $31 \%$ & 0.0011 & $3.26 \sigma$ \\
$10^{21}$ & $21 / 43$ & $49 \%$ & 0.50 & $0.67 \sigma$ & $10 / 41$ & $24 \%$ & 0.00073 & $3.38 \sigma$ \\
$10^{22}$ & $25 / 53$ & $47 \%$ & 0.39 & $0.86 \sigma$ & $6 / 31$ & $19 \%$ & 0.00044 & $3.52 \sigma$ \\
$10^{23}$ & $31 / 67$ & $46 \%$ & 0.31 & $1.00 \sigma$ & $0 / 17$ & $0 \%$ & $7.6 \times 10^{-6}$ & $4.46 \sigma$ \\
\hline
\end{tabular}

NotE. $-k$ is the number of 21 -cm detections below/above the partition and $n$ is the total number of sources in the same region, "rate" expresses this as a percentage detection rate, $P(\leq k / n)$ is the binomial probability of this number of detections or less occuring by chance for an unbiased sample and $S(\leq k / n)$ is the significance of this (derived assuming Gaussian statistics). 
Since, in the optically thin regime, the column density of the neutral gas is related to the 21-cm absorption strength via $N_{\mathrm{HI}} \propto\left(T_{\mathrm{s}} / f\right) . \int \tau d v$, where $T_{\mathrm{s}}$ is the spin temperature of the absorbing gas and $f$ the covering factor of the background flux, a possible cause of the nondetections at high redshift could be elevated spin temperatures, a trait which may have been observed in intervening absorption systems (Kanekar \& Chengalur 2003). However, for these, the mean value at $z_{\text {abs }} \gtrsim 1$ may only be double that at $z_{\text {abs }} \lesssim 1\left(T_{\text {spin }} / f=2400 \mathrm{~K}\right.$, cf. $1200 \mathrm{~K}$, Curran et al. 2010), a factor which can be accounted for by the different line-of-sight geometry effects, introduced by a flat expanding Universe (Curran \& Webb 2006). This could negate the need for significantly higher spin temperatures in order to explain the lower incidence of detections at high redshift.

In the case of associated absorption, with $z_{\mathrm{abs}} \approx z_{\mathrm{em}}$ the same geometry effects cannot be responsible for such a discrepancy between the low and high redshift samples, although the generally higher radio luminosities of the latter could be raising the spin temperature through a high population of the upper hyperfine level. We have already suggested that the more intense ultra-violet fluxes could be responsible for the deficit in detections at high redshift (Sect. 2.1.1), which could be causing large ionisation fractions and/or a raising of the spin temperature (Field 1959; Bahcall \& Ekers 1969) and so in Fig. 3 we show the ultra-violet luminosity (sourceframe $\lambda \approx 1216 \AA$ ) versus that of the radio (source-frame $\lambda \approx 21 \mathrm{~cm})$.

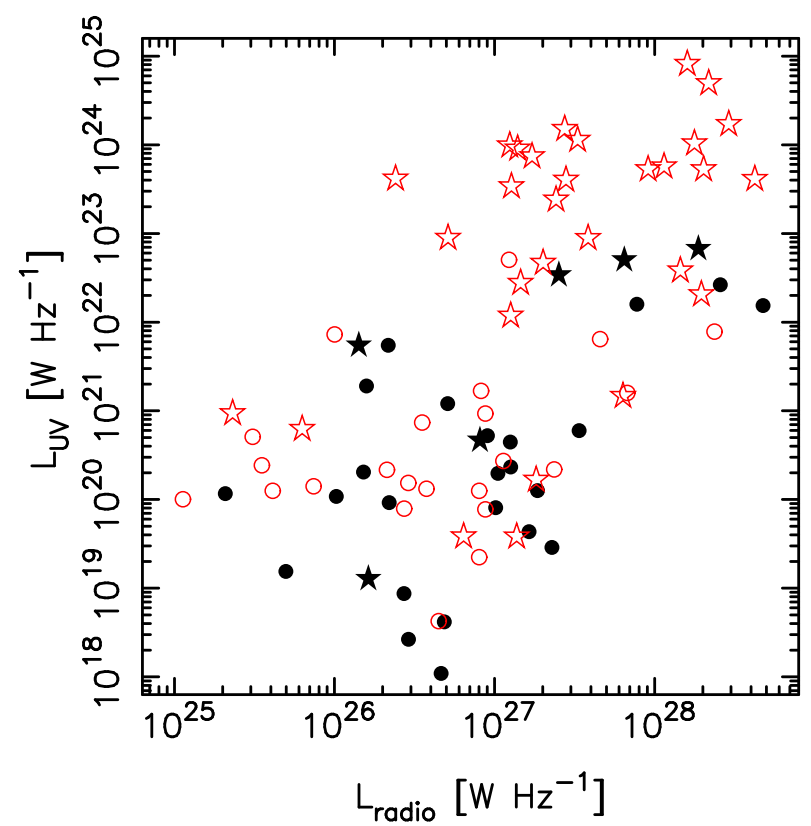

FIG. 3.- The ultra-violet versus the radio luminosity for the sample. Again, the filled symbols represent the $21-\mathrm{cm}$ detections and the unfilled symbols the non-detections, with stars signifying quasars and circles galaxies (see Sect. 2.3).

There is a strong apparent correlation between the two luminosities $(5.27 \sigma)$, with a large dispersion, particularly in the UV luminosity. Any relationship will, however, be largely driven by both quantities having a strong redshift dependence. However, we are not sensitive to sources in the bottom right corner, where the optical flux is too low to obtain a redshift (all sources in this sample are identified as having associated absorption). Also, while all of the $21-\mathrm{cm}$ detections are limited to $L_{\mathrm{UV}} \lesssim 10^{23} \mathrm{~W}$ $\mathrm{Hz}^{-1}$, they do cover the same range of radio luminosities as the non-detections, thus suggesting that these are not so critical in the detection of 21-cm absorption. As a check, we apply Equ. 1 to the radio luminosities and find no significant relationship between the detection rate and the radio continuum luminosity (Fig. 2, right). This is in contrast to the same statistic applied to the ultraviolet luminosities and so it appears unlikely that the radio power is the dominant effect in raising the spin temperature of the gas above the detection thresholds.

\subsection{Source structure and environment}

\subsubsection{Host galaxy environments}

In considering the presence or otherwise of absorbing neutral gas, one needs to consider the wider picture of the quasar host galaxy and its environment. A great deal is known about the host galaxies of luminous AGN at low redshift. Imaging studies, particularly with HST, of $z \lesssim 0.4$ quasars (Tavlor et al. 1996b; Bahcall et al. 1997; Dunlop et al. 2003; Flovd et al. 2004), indicate that early-type or spheroidal hosts are much more likely for high-luminosity quasars, and that almost all luminous radio-loud quasars and radio galaxies are to be found in elliptical galaxies.

Studies at higher redshift, often with ground-based adaptive optics (Hutchings et al. 1999; Kukula et al. 2001; Ridgway et al. 2001; Peng et al. 2006) show a slightly more complex picture. The luminous quasars tend to follow the same trend, with elliptical/early-type hosts predominant, although it is more difficult to gain a confident picture of the host morphology at these redshifts. Using gravitationally-lensed quasars can provide some extra resolution, from which Peng et al. (2006) find that quasar hosts at $1<z<4.5$ span a range of morphologies from early-type to disky/late-type galaxies.

Determining the expected $\mathrm{H}$ I content of typical quasar host galaxies is difficult, as 21-cm absorption is often the only way to probe the neutral gas at such redshifts. For low redshifts, $21-\mathrm{cm}$ emission studies are possible with much being gleaned from blind surveys: The HIPASS survey had detection rates of $6 \%$ for RC3 ellipticals and $13 \%$ for S0s (Sadler et al. 2002) ${ }^{4}$ and from the ALFALFA survey, di Serego Alighieri et al. (2007) detected $\mathrm{H}$ I in just 2-3\% of bright early-type galaxies in the Virgo cluster, with only one of these (M86, an S0) having $M_{\mathrm{B}}<-20\left(L_{\mathrm{B}} \gtrsim 2 \times 10^{22} \mathrm{~W} \mathrm{~Hz}^{-1}\right)$. Grossi et al. (2009), however, examined early-type galaxies in low-density environments, and found a higher detection fraction of $\mathrm{H} \mathrm{I}$ emission for the more luminous objects, although neither of the two ellipticals with $M_{\mathrm{B}}<-20$ were detected.

Targeted searches with interferometers (Oosterloo et al. 2002, 2007; Morganti et al. 2006; Nyland et al. 2009) show different results. The detection fraction tends to be much larger than in the blind surveys, with a mix of ordered, relaxed disks and more complex interacting systems seen. However, these have mostly been limited to fairly local galaxies, although a small number of more powerful (yet still nearby

4 Although $30-50 \%$ of these were confused, with more than one optical galaxy in the beam. 
compared to our sample, $z<0.03)$ radio galaxies have been observed to have $\mathrm{H}$ I disks as well as tails, resulting from an interaction, which often coincide with $\mathrm{HI}$ absorption. One such example is NGC612 Emonts et al. 2008), an S0 galaxy with a large neutral disk of gas, likely originating in an interaction or merger. However, hosts luminous AGN are very rare in the local universe, making a clear understanding of the $\mathrm{HI}$ properties elusive.

The above can provide qualitative description of why we see the cut-off in the absorption distribution at high luminosities, where the the hosts tend to be the larger elliptical galaxies. For instance, the lowredshift luminous AGN imaged by the HST all have elliptical hosts (Bahcall et al. 1997; Dunlop et al. 2003; Floyd et al. 2004) and $M_{\mathrm{V}} \lesssim-23$, which corresponds to a $V$-band luminosity of $L_{\mathrm{V}} \gtrsim 10^{22} \mathrm{~W} \mathrm{~Hz}^{-1}-$ similar to the brightest sources in our sample. Regarding the $L_{\mathrm{V}} \gtrsim 10^{23} \mathrm{~W} \mathrm{~Hz}^{-1}$ sources of our sample, of the five imaged, two have resolved host galaxies both of which are ellipticals (J1540+1447; Urry et al. 2000 and J2250+1419; Dunlop et al. 2003) $)^{5}$.

To summarise, at lower luminosities the observed morphological mix could present a range of H I column densities, whereas at higher luminosities the hosts are expected to be gas-poor ellipticals, resulting in a lack of $21-\mathrm{cm}$ absorption. This could give a mix of $21-\mathrm{cm}$ detections at low redshift and exclusive non-detections at high redshift (Fig. 11). However, the bulk of the $z \geq 0.1$ sample has not been observed with a resolution sufficient to determine the host galaxy morphologies, so that, while we do have a good understanding of how the 21-cm detections are distributed with $L_{\mathrm{UV}}$, how these are distributed with morphology for this sample is at present undetermined.

\subsubsection{Radio source sizes}

In addition to any possible evolution in the host galaxy environments, selection effects may arise from the large range of beam sizes and radio source sizes over what constitutes a heterogeneous sample (all radio sources at $z \geq 0.1$ searched for 21-cm absorption). For example, a $10^{\prime \prime}$ diameter beam (a typical lower value for the GMRT at 90-cm, Curran et al. 2008c) covers a linear extent of $18 \mathrm{kpc}$ at $z=0.1, \approx 86 \mathrm{kpc}$ (the maximum) at $z=1.6$ and $63 \mathrm{kpc}$ at $z=5.2$. That is, the area subtended by a given telescope beam can vary by a factor of $\approx 20$ due to the redshift range alone, an effect which is compounded by the published searches being performed with numerous instruments/configurations, each with its own beam size. This can, in principle, affect the sensitivity to 21$\mathrm{cm}$ absorption. For instance, absorption can be missed in near-by Seyfert galaxies by lower resolution observations, while being revealed on VLBA/MERLIN scales (e.g. Mundell et al. 2003).

Therefore, the radio source sizes and the bias these may have on the effective coverage of the background emission must be considered. This, however, is fraught with uncertainties as the heterogeneity of the sample

\footnotetext{
${ }^{5}$ Of the remaining three, J0201-1132 and J1829+4844 are only marginally resolved (Kotilainen \& Falomo 2000; Lehnert et al. 1999, respectively) and in J0927+3902 no host is seen (Carballo et al. 1998).
}

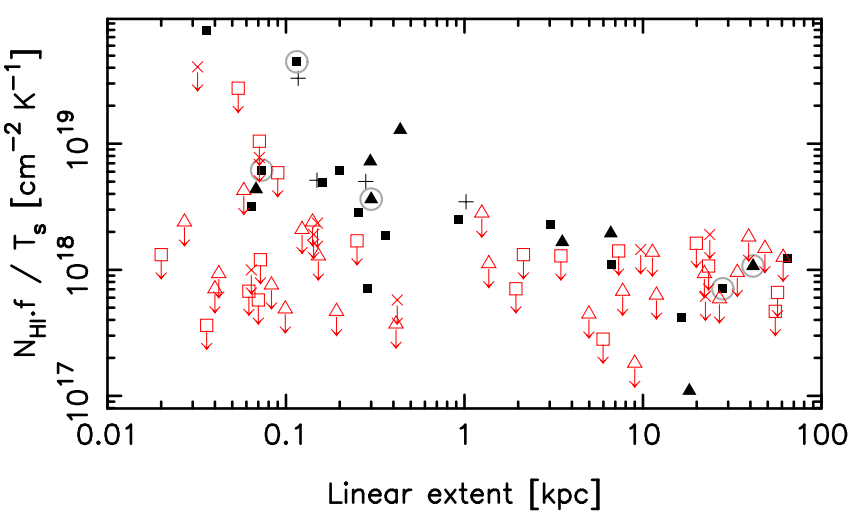

FIG. 4.- The scaled velocity integrated optical depth $(1.823 \times$ $\left.10^{18} \cdot \int \tau d v\right)$ of the $21-\mathrm{cm}$ absorption versus the linear extent of the radio source size (from the references listed in Tables 1 and 2). The symbols are as per Fig. 1 where the downwards arrows signify the upper limits to the non-detections. The five detections not classified as compact objects (CSO, CSS, GPS nor HFP, defined in Sect. 2.4) are circled.

combined with the very different scales probed, will contribute to diluting out any strong trends in the linear extents of the sources. Further compounding this is the fact that the radio emission can exhibit very different structure at different frequencies and, although the sizes used are from the nearest available frequencies,VLBI and VLBA continuum observations are typically at $\gtrsim 2 \mathrm{GHz}$, significantly higher than that of the redshifted $21-\mathrm{cm}$ line. The only way to fully address this would be through dedicated mapping of the 21-cm absorption (e.g. Lane et al. 2000; Mundell et al. 2003), although the available VLBA bands would only allow this at redshifts of $1.27 \leq z \leq 1.38$ and $3.15 \leq z \leq 3.55$, thus covering only six of the sample (all of which are non-detections in any case).

Nevertheless, applying these source sizes, in Fig. 4 we see the same "column density" (actually the velocity integrated optical depth)-linear size anti-correlation reported for the GPS and CSS sources by Pihlström et al. (2003); Gupta et al. (2006): A Kendall's $\tau$-test on the detections gives a two-sided probability $P(\tau)=1.82 \times 10^{-5}$ of the correlation arising by chance, which is significant at $4.28 \sigma$ assuming Gaussian statistics. Including the 21$\mathrm{cm}$ non-detections, through the ASURV survival analysis package (Isobe et al. 1986), decreases this to $P(\tau)=$ $0.017(2.39 \sigma)$. If we consider just the GPS and CSS sources (Pihlström et al. 2003$)^{6}$, we obtain $P(\tau)=0.006$ $(2.75 \sigma)$, which nonetheless indicates that the inclusion of the non-detections significantly worsens the correlation. This suggests that the 21-cm non-detections may be subject to an additional effect. This could be due to smaller absorption cross-sections further reducing the covering factor, generally higher spin temperatures and/or lower neutral hydrogen column densities, all of which could be due to both orientation and UV luminosity effects.

Although the decrease in the "column density" is most likely due to a decrease in the covering factor with increasing with linear extent, there is also the possibility

\footnotetext{
${ }^{6}$ Although from Fig. 4 4 it appears that all of the detections follow the same $\int \tau d v$-linear size correlation as is seen for the compact objects only (Pihlström et al. [2003; Gupta et al. 2006), although this is based upon the small number of non-compact objects (five).
} 
TABLE 4

The MEAN ULTRA-VIOLET LUMINOSITIES $\left[\mathrm{W} \mathrm{Hz}^{-1}\right]$ FOR THE GALAXY AND QUASAR SUB-SAMPLES. $\sigma$ GIVES THE STANDARD DEVIATION.

\begin{tabular}{lcccc}
\hline & \multicolumn{2}{c}{ GALAXIES } & \multicolumn{2}{c}{ QUASARS } \\
& Detections & All & Detections & All \\
\hline $\log _{10} L_{\mathrm{UV}}$ & 20.3 & 20.4 & 21.6 & 22.7 \\
$\sigma$ of $\log _{10} L_{\mathrm{UV}}$ & 1.1 & 1.1 & 1.3 & 1.5 \\
Sample size & 25 & 48 & 6 & 36 \\
\hline
\end{tabular}

that the 21-cm absorption towards the larger sources is more susceptible to dilution by the extended $21-\mathrm{cm}$ emission. However, in spite of the four orders of magnitude span in size, we find no significant difference in the detection rates between the smaller $(<1 \mathrm{kpc}, 16$ out of 42$)$ and the larger ( $\geq 1 \mathrm{kpc}, 11$ out of 36$)$ sources (Fig. (4), indicating that this is not a strong effect.

\subsection{AGN spectral type and source classification}

If the 21-cm non-detections are due to orientation effects, it would suggest that all of the $L_{\mathrm{UV}} \gtrsim 10^{23} \mathrm{~W} \mathrm{~Hz}^{-1}$ sources are type- 1 objects and therefore the gas may only necessarily be ionised along our line-of-sight, which is direct to the AGN. To examine this question, we make use of the spectral classifications obtained by Curran et al. (2008c). These were compiled by exhaustively searching the literature for published emission-line fluxes or spectra. Sources with broad permitted lines were classified as type- 1 and those with only narrow lines as type-2. Our classifications generally agree with those of Vermeulen et al. (2003), except for $2316+0405$ which we assign as type-2 (Miller 1981), although Vermeulen et al. (2003) have this classified as a broad line radio galaxy.

Using these classifications, it is apparent that all the high UV luminosity sources are indeed type-1 (Fig:1). However, they appear to be distinct from the population of lower UV luminous type- 1 objects, some of which have been detected in 21-cm absorption at, somewhat surprisingly, the same detection rate $(50 \%)$ as for the type-2 objects. That is, the overall bias towards type- 1 nondetections is caused by the inclusion of the $L_{\mathrm{UV}} \gtrsim 10^{23}$ $\mathrm{W} \mathrm{Hz}^{-1}$ non-detections. Therefore, the ultra-violet luminosity of the object appears to have much more bearing on whether 21-cm absorption can be detected and, at moderate UV luminosities, the AGN type provides no indicator of whether a high column of cold neutral gas is likely to intercept our sight-line.

Nevertheless, as has been noted in the literature (e.g. Pihlström et al. 2003; Gupta \& Saikia 2006b), 21-cm absorption is more likely to be detected in a radio galaxy than in a quasar, even if there are even odds between the two AGN types. We designate each object in our sample as either a quasar or a galaxy, using the classifications from Curran et al. (2008c). To obtain these, optical imaging and spectroscopy from the literature were used to distinguish sources dominated by the nuclear source (the "quasars") from those dominated by the extended stellar light of the host galaxy (the "galaxies"), the latter being either weaker AGN or relatively obscured type-2 AGN.

From Table 4, where we show the average UV luminosities for each class, we see that there is little difference in the luminosities between the detections and whole sample for the galaxies (as well as a 50\% detection rate). However, the quasars in which 21-cm has been detected are generally an order of magnitude brighter than the galaxies, with the whole quasar sample being another order of magnitude brighter yet (with only a 17\% detection rate). Again, over and above an underlying $50 \%$ due to orientation effects, this strongly suggests that it is the ultra-violet luminosity which is the key criterion in the detection of $\mathrm{HI}$ in these objects and that any detection bias against the quasars is due to their generally higher ultra-violet output.

\subsection{Detection rates in compact objects}

Until the $725-1200 \mathrm{MHz}$ survey of Vermeulen et al. (2003), there were few detections of associated 21-cm absorption at $z \geq 0.1$. Of the Vermeulen et al. (2003) detections most (17 out of 19) are gigahertz peaked spectrum and compact steep spectrum sources, with the general consensus being that these exhibit higher $21-\mathrm{cm}$ detection rates than other radio sources, due to their gas rich-host galaxies (Conwav 1997; O'Dea 1998; Gupta et al. 2006 and references therein). CSSs and GPSs are believed to be intrinsically small $(\lesssim 10$ and $\lesssim 1 \mathrm{kpc}$, respectively) and may be the young precursors of the typically large radio sources, themselves evolving from compact symmetric objects (CSOs, Fanti et al. 1995). Although the high occurrence of broad forbidden lines may suggest that these are primarily type-1 objects, in which the compact appearance is due to the radio lobes being directed along our sight-line, the diminished sizes are not believed to be due to projection effects (Fanti et al. 1990): Although there are radio lobes present, the jets may be embedded in a dusty interstellar medium so that the AGN is believed to be subject to significant extinction (e.g. Bellamy et al. 2003), resulting in strong radio emission as the trapped jets interact with the rich, dense cocoon, in this early evolutionary stage.

The possibility that CSSs and GPSs are compact type1 objects, would be consistent with the 21-cm absorption being, on average, blue-shifted with respect to the optical redshift (Vermeulen et al. 2003, see Sect. 3.1.2). Furthermore, the efficient coverage of the confined radio core could contribute to a high incidence of $21-\mathrm{cm}$ absorption, which probably occurs in an outflow. However, of the 17 CSS/GPS detections of Vermeulen et al. (2003) only six are classified as type- 1 objects, cf. nine type-2s (and two unclassified, Table 1), which again may suggest that these objects have random orientations, with the possibility of absorption arising in either an outflow or the disk.

If 21-cm absorption favours compact objects (and this was a more important effect than the UV luminosity), we may expect a very low number of CSSs/GPSs in the exclusively non-detected $L_{\mathrm{UV}} \gtrsim 10^{23} \mathrm{~W} \mathrm{~Hz}^{-1}$ sample. However, three of the eight low redshift $L_{\mathrm{UV}} \gtrsim 10^{23} \mathrm{~W}$ $\mathrm{Hz}^{-1}$ objects of Vermeulen et al. (2003) are classified as CSS/GPS, and so these are not immune to the 21-cm absorption being undetected at high UV luminosity. Being from the Parkes Quarter-Jansky Flat-spectrum Sample, the high redshift sources are, by definition, flat spectrum (with $\alpha>-0.5$ ), although of these eight the radio SEDs for 1351-018 and 1535+004 are suggestive of 


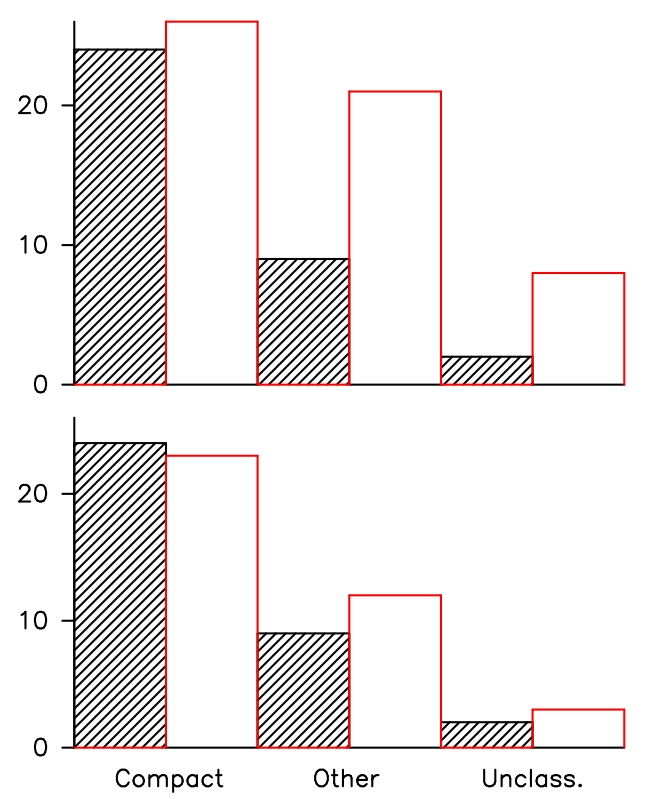

FIG. 5.- The incidence of detections (hatched histogram) and non-detections (unfilled histogram) for compact objects (CSO, CSS, GPS and HFP) compared to the others. Top - the whole sample. Bottom - those with $L_{\mathrm{UV}} \leq 10^{23} \mathrm{~W} \mathrm{~Hz}^{-1}$.

GPSs or high frequency peaker galaxies $(\mathrm{HFPs})^{7}$. For the remainder of the $L_{\mathrm{UV}} \gtrsim 10^{23} \mathrm{~W} \mathrm{~Hz}^{-1}$ sample, however, being flat spectrum sources (de Waard et al. 1985; Jackson et al. 2002; Vermeulen et al. 2003) may also suggest that many of these are oriented end-on with respect to us, consistent with their type-1 status (Sect. 2.3).

Although the vast majority of the detections of Vermeulen et al. (2003) are CSS/GPS, 22 of the 38 nondetections are also classified as such (giving a CSS/GPS detection rate of $44 \%$ ). Furthermore, Pihlström et al. (2003) and Gupta \& Saikia (2006b) both find a $\approx 50 \%$ detection rate in their CSS/GPS samples, as well as the $33 \%$ rate from the (albeit small) HFP sample of Orienti et al. (2006). Summarising this in Fig. 5 (top), we find the overall detection rate to be $47 \%$, cf. $30 \%$ for the "others", not classified as CSO/CSS/GPS/HFP, and $20 \%$ for those unclassified, thus indicating higher detection rates in compact objects.

This distribution, however, becomes more uniform (51\% - compact, $43 \%$ - others \& $40 \%$ - unclassified) between the classes when considering the $L_{\mathrm{UV}} \leq 10^{23} \mathrm{~W}$ $\mathrm{Hz}^{-1}$ sources only (Fig. [5 bottom). That is, the compact source detection rate may not be significantly higher than that of the others in the moderate UV luminosity sample and, again, it is the inclusion of $L_{\mathrm{UV}} \gtrsim 10^{23} \mathrm{~W}$

\footnotetext{
7 The turnover frequencies of $\gtrsim 5 \mathrm{GHz}$ may suggest newly born radio sources (Dallacasa 2003) and from the anti-correlation between turnover frequency and the source size (Fanti et al. 1990; O'Dea \& Baum 1997), we also expect these to be extremely compact. For the GPS/HFP suspects of the $L_{\mathrm{UV}} \gtrsim 10^{23} \mathrm{~W} \mathrm{~Hz}^{-1}$ targets of Curran et al. (2008c), the Very Large Array's FIRST (Faint Images of the Radio Sky at Twenty Centimetres, White et al.1997) survey gives deconvolved source sizes of $<0.99^{\prime \prime} \times 0.39^{\prime \prime}$ for 1351 018 and $<1.18^{\prime \prime} \times 0.76^{\prime \prime}$ at an observed frequency of $1.4 \mathrm{GHz}$. At redshifts of $z=3.707$ and 3.497 , these sizes correspond to $<7 \times 3$ $\mathrm{kpc}$ and $<9 \times 6 \mathrm{kpc}$. respectively, where at a turnover frequency of $\sim 10 \mathrm{GHz}$, we may expect a source size of $\lesssim 1 \mathrm{kpc}$ (O'Dea \& Baum 1997).
}

TABLE 5

THE MEAN ULTRA-VIOLET LUMinOSities $\left[\mathrm{W} \mathrm{Hz}^{-1}\right.$ ] AND GALAXY/QUASAR DISTRIBUTION FOR THE SAMPLE BASED UPON RADIO CLASSIFICATION.

\begin{tabular}{lccc}
\hline & Compact & Other & Unclassified \\
\hline $\log _{10} L_{\mathrm{UV}}$ & 20.9 & 21.7 & 22.4 \\
$\sigma$ of $\log _{10} L_{\mathrm{UV}}$ & 1.4 & 1.7 & 2.0 \\
No. galaxies & 35 & 15 & 4 \\
No. quasars & 15 & 15 & 6 \\
Mean redshift $\pm 1 \sigma$ & $0.60 \pm 0.96$ & $0.67 \pm 0.78$ & $2.5 \pm 1.4$ \\
\hline
\end{tabular}

$\mathrm{Hz}^{-1}$ sources which introduces a bias ${ }^{8}$. This possibility is supported by the average UV luminosities of the radio classes, which are appreciably lower for the compact objects (Table 5), which could be consistent with these being young sources in which the AGN activity (radio and ultra-violet) has yet to reach its full strength (Fig. 3). Therefore, it is possible that compact objects exhibit these higher $21-\mathrm{cm}$ detection rates due mainly to their generally low UV luminosities, with the line strength in these being dominated by effect of the projection of the radio lobes on the covering factor (Sect. 2.2.2).

\section{THE LOCATION OF THE ABSORBING GAS}

\subsection{H I 21-cm line kinematics}

\subsubsection{Absorption in the galactic disk}

For the moderate UV luminous sample, which are not expected to be dominated by elliptical hosts (Sect. 2.2.1), the 50\% detection rate for both type- 1 and type-2 objects at $L_{\mathrm{UV}} \lesssim 10^{23} \mathrm{~W} \mathrm{~Hz}^{-1}$ strongly suggests that the absorption does not occur in the obscuring torus associated with the AGN. The next logical candidate is therefore the large-scale galactic disk, where most of the gas would be expected to reside, as is seen in absorption studies of low redshift starburst and Seyfert galaxies. In particular, Gallimore et al. (1999) find that the 21-cm line strength is correlated with the galaxy inclination in $z \leq 0.04$ Seyferts. Furthermore, although they suggest that the incidence of 21-cm absorption is broadly consistent with unified schemes of AGN, Morganti et al. (2001) acknowledge that it is likely that some absorption is also occuring beyond the sub-pc scale of the circumnuclear torus. Although we may expect the gas to be nearly coplanar on all scales (e.g. Curran et al. 2008a), if the large-scale disk is the source of the absorption, the fact that both types of AGN have the same odds of exhibiting 21-cm absorption would suggest that the orientation of the large-scale disk with respect to the small-scale obscuring torus is random.

At redshifts of $z \geq 0.1$, the disk orientations cannot generally be determined, although from lower redshift Seyfert studies we can show the inclination of the galaxy disk for various Seyfert types (Fig. 6). From the figure we see the full range of possible offsets between the position angles of the radio jets and the position angle of the host galaxy (top panel). Were the obscuring torus and main galaxy disk coplanar, we would expect the points on the ordinate to be concentrated close to

8 Although a larger number of the non-compact objects have luminosities of $L_{\mathrm{UV}} \leq 10^{23} \mathrm{~W} \mathrm{~Hz}{ }^{-1}$, note that there are several CSSs/GPSs close to the UV cut-off (Table 2). 


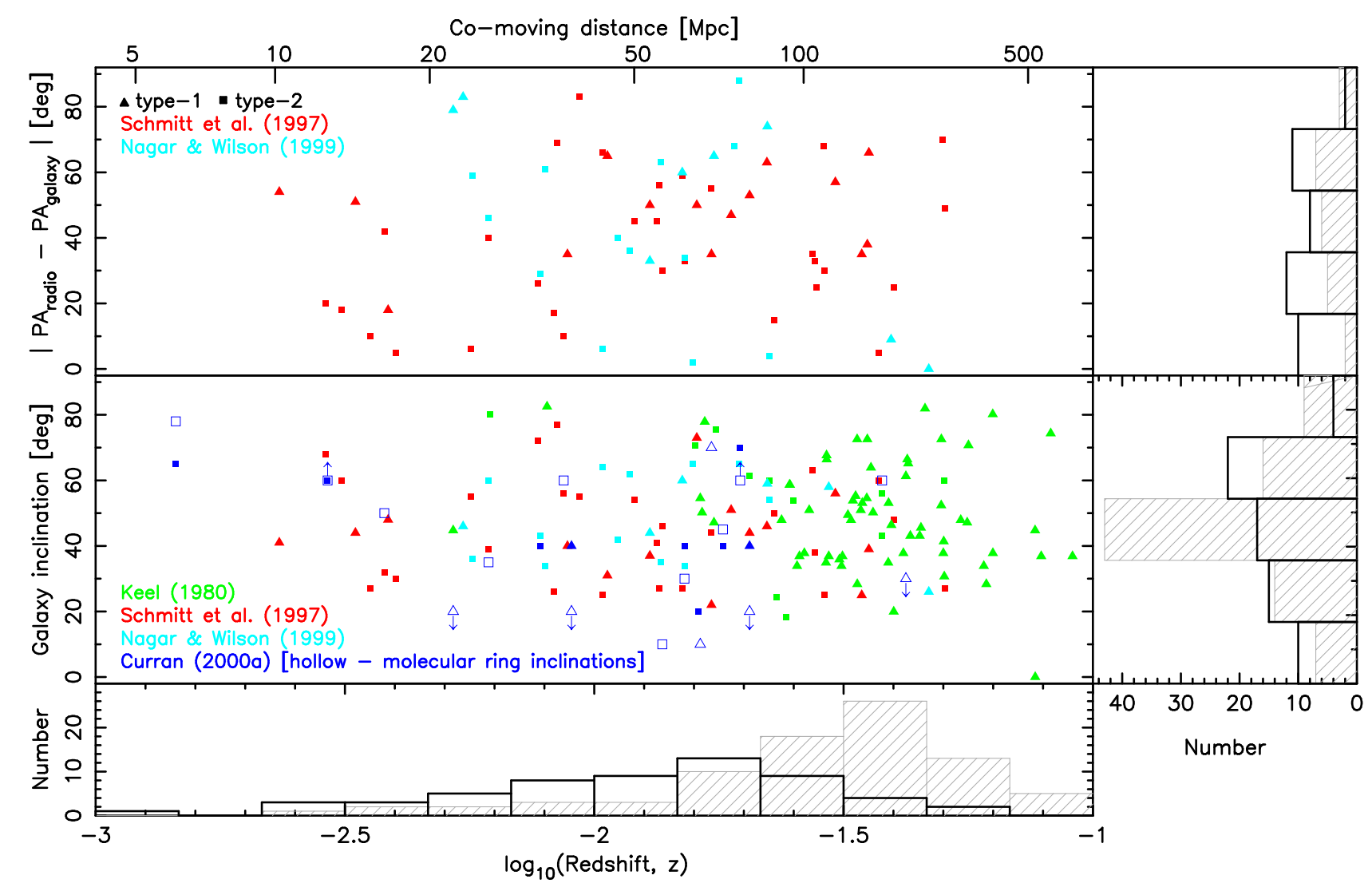

FIG. 6. - The difference in the position angles of the radio and dis axes (top) and the inclinations of the galaxies (bottom) hosting low redshift Seyferts. The triangles/hatched histogram represent the type-1 objects and the squares/unfilled histogram the type-2, with the colours of the symbols indicating the source reference (Keel 1980; Schmitt et al. 1997; Nagar \& Wilson 1999; Curran 2000a).

$0^{\circ}$ for both AGN types, but, as noted by Schmitt et al. (1997); Nagar \& Wilson (1999), the distribution tends to be quite random. Furthermore, if the torus is coplanar with the host disk, we would expect type-1 Seyferts to occupy galaxies of low inclination and type-2s in those of high inclination. However, from the vertical histogram (Fig. 6, bottom panel) it is apparent that neither Seyfert type has a preferred disk inclination, with both types exhibiting a mean inclination of $48^{\circ}\left(\sigma \approx 16^{\circ}\right.$, from 83 exclusive $^{9}$ type- 1 objects and 58 exclusive type- $2 \mathrm{~s}$ ). Interestingly, although the large-scale disk exhibits a random orientation with respect to the circumnuclear torus, the sub-kpc molecular ring is generally aligned (the hollow symbols in Fig. 6): Although the numbers are much smaller, the mean inclination of the molecular ring in the six type- 1 objects is $<28^{\circ}$, cf. $>49^{\circ}$ for the ten type- 2 objects $\left(\sigma \approx 19^{\circ}\right.$, with the limits being due to limits in the inclination estimates, Curran 2000a). A Kolmogorov-Smirnov test gives a $<6 \%$ probability that the inclinations of the type- 1 and type- 2 molecular rings are drawn from the same sample, in contrast to $38 \%$ for the galactic disk inclinations. This alignment between the molecular ring and torus may be expected, despite the random larger scale orientations, as these rings generally only reach $\sim 1 / 100$ th the extent of the atomic gas (Mvers \& Scoville 1987; Plante et al. 1991; Bergman et al. 1992; Irwin \& Sofue 1992; Tacconi et al.

9 Sources common to more than one sample have only been counted once.
1994; Kohno et al. 1996; Curran et al. 1998; Israel et al. 1998) $)^{10}$ and may be funneling the material to the smaller scale torus (see Curran 2000b and references therein) ${ }^{11}$.

\subsubsection{Absorption due to outflows}

Aside from the disk, as noted above, absorption may also be due to in-falling gas or outflows. That is, gas located along the polar axes, thus being located between us and the AGN in type- 1 objects, rendering the gas detectable in absorption. Since this gas may exhibit a wider profile (FWHM) than gas tracing the rotation of the disk ${ }^{12}$, while having a larger offset from the systemic velocity of the galaxy $(\Delta v)$, we may expect type-1 objects to be grouped separately from type-2s in a plot of FWHM versus $\Delta v$, at least in terms of the abscissa.

Showing this in Fig. [7 we see no clear distinction between the AGN types in either axis. For the FWHM, the type-1 absorbers do exhibit slightly wider profiles, which would suggest that the outflows are subject to large ve-

10 Although the molecular gas beyond the ring can be seen to extend much farther (e.g. Young \& Scoville 1991; Curran et al. 2008a).

11 http://nedwww.ipac.caltech.edu/level5/Curran/frames.html

12 For example, presuming the systemic velocities are sufficiently accurate to determine these large blue-shifted offsets, outflows with widths of $\gtrsim 1000 \mathrm{~km} \mathrm{~s}^{-1}$ are seen in some low redshift radio galaxies (Morganti et al. 2005b, 2007), in addition to the broad components observed by Morganti et al. (2003, 2005a), in which the blue-shifts from the peak absorption component are strongly suggestive of outflows. 


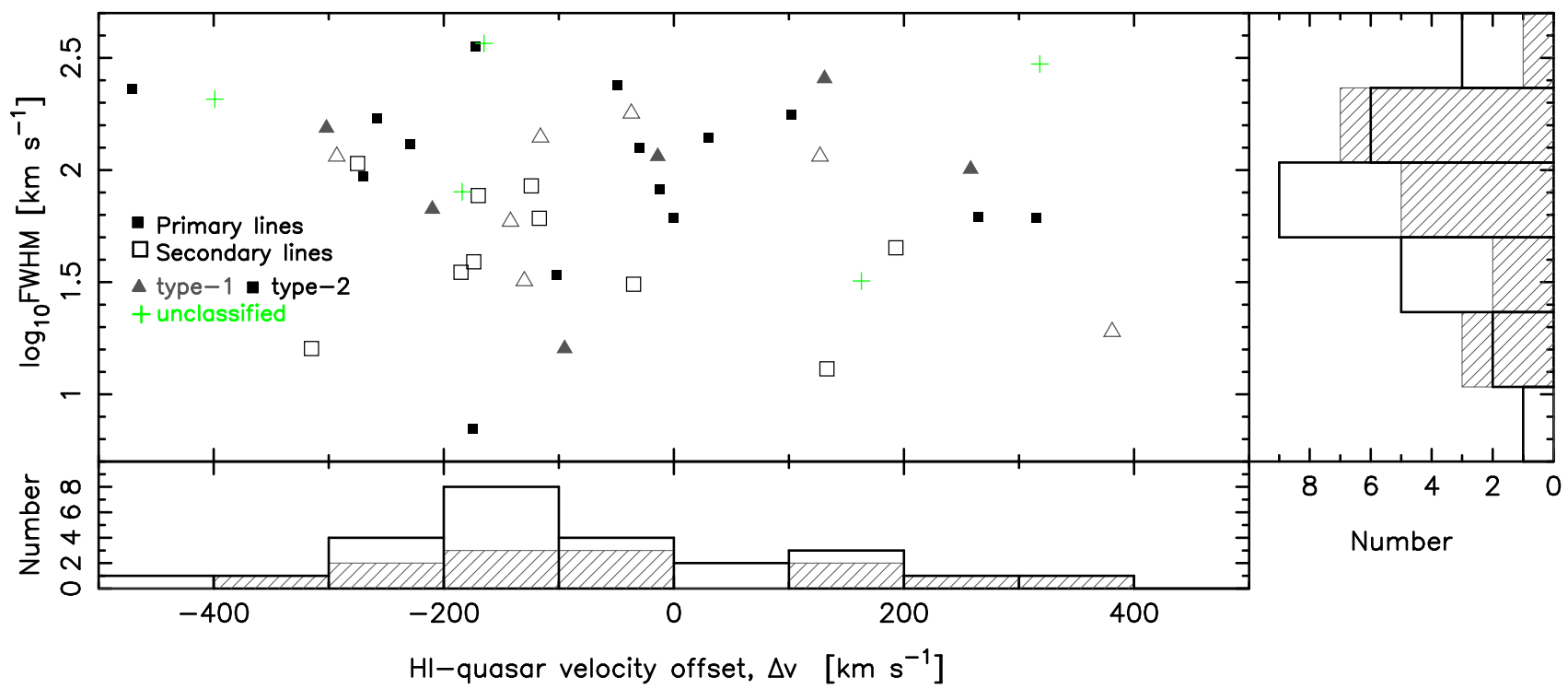

FIG. 7.- The profile width versus the offset from the systemic velocity for the 21-cm detections at $z \geq 0.1$. The triangles/hatched histogram represent the type-1 AGN and the squares/unfilled histogram the type-2. + signifies that there is no AGN classification available. We have plotted each individually resolved absorption component and flagged the primary (filled symbols) and secondary (unfilled) absorption lines, where the primary is the line with the largest optical depth and the secondaries are the remaining shallower lines (see Vermeulen et al. 2003). For the sake of clarity, the plot has been truncated to $|\Delta v| \leq 500 \mathrm{~km} \mathrm{~s}^{-1}$, although there are five cases with $\Delta v<-500 \mathrm{~km} \mathrm{~s}^{-1}: 1413+135$ at $-705 \mathrm{~km} \mathrm{~s}^{-1}$, J1815+6127 at $-1258 \mathrm{~km} \mathrm{~s}^{-1}$, J1821+3942 at $-869 \mathrm{~km} \mathrm{~s}^{-1}$ (primary lines), as well as the $-742 \mathrm{~km} \mathrm{~s}^{-1}$ secondary line in the latter object. All of these lines arise in type- 1 objects, with the one type- 2 being in J1944+5448 with $-1420 \mathrm{~km} \mathrm{~s}^{-1}$ (primary line). On the redshifted end there are just three cases at $v>500 \mathrm{~km} \mathrm{~s}^{-1}$ - the two unclassified AGN J0431+2037 at $+636 \mathrm{~km} \mathrm{~s}^{-1}$ and $0902+343$ at $+970 \mathrm{~km} \mathrm{~s}^{-1}$, as well as the type-1 object $1549-79$ at $+665 \mathrm{~km} \mathrm{~s}^{-1}$.

locity differentials, as has been seen in several low redshift cases (e.g. Morganti et al. 2003, 2005a,b, 2007). If absorption were occuring in the circumnuclear torus, we may also expect very wide profiles for the type-2 AGN, possibly much wider than those of the galactic disk itself ${ }^{13}$. As it is, we can make no distinction between the FWHM of the type- 1 and type- 2 objects and unlike in emission, the absorption profile widths will ultimately be subject to the covering factor and the size of the continuum source, making any distinction between disk and outflow absorption difficult.

Again, for the velocity offsets there is no real difference between the two AGN types and both exhibit a slight bias towards blue-shifted absorption ${ }^{14}$. Although uncertainties of $\sim 10^{2} \mathrm{~km} \mathrm{~s}^{-1}$ due to the optical emission lines in $\Delta v$ are to be expected, many studies show a tendency for the absorption to be blue-shifted with respect to the systemic velocity (Vermeulen et al. 2003; Morganti et al. 2003, 2005a, b, 2007). If these offsets are artifacts of poorly constrained optical redshifts, we would expect similar numbers of red-shifted components and, as stated previously, in low redshift radio galaxies wide blue-shifted tails are seen in the profiles, where the main feature is close to the systemic velocity (Morganti et al. 2003, 2005a).

13 For example, in the type-2 Seyfert NGC 4258, the subparsec disk is found to have a rotation speed of $900 \mathrm{~km} \mathrm{~s}^{-1}$ (Haschick et al. 1996) and in the Circinus galaxy, also a type-2 Seyfert, the $\mathrm{H}_{2} \mathrm{O}$ masers also trace a disk which rotates much more rapidly than the galactic disk (Greenhill et al. 2003, cf. Curran et al. 2008a).

14 This is most obvious for the type-2s in Fig. 7 but as stated in the caption, there are also four (three primary and one secondary) type- 1 absorbers with $\Delta v<-500 \mathrm{~km} \mathrm{~s}^{-1}$.
Since many of the $21-\mathrm{cm}$ detections at $z \geq 0.1$ are from Vermeulen et al. (2003) [Table 1, it is not surprising that we also see this skew towards blue-shifted absorption (Fig. 7). Therefore in Table 6, where we show the average offsets for the AGN classes, we also show the contribution from the remainder of the literature.

As seen from the table, although the standard deviations are large, the additional results confirm that on average the absorption is blue-shifted, which could indicate outflows or some other non-symmetric mechanism as the origin. The statistics also confirm the larger spread in the velocity offsets of the type-1 AGN, which is not wholly evident from Fig. 7, due to the three (primary) type- 1 absorbers offset at $\Delta v<-500 \mathrm{~km} \mathrm{~s}^{-1}$. If it were just these three at the blue-shift extremes, we could at least state that some type-1s show more of a bias for absorption in rapidly outflowing gas, although there is the type-2 case $(\mathrm{J} 1944+5448)$ with $\Delta v=-1420 \mathrm{~km} \mathrm{~s}^{-1}$. This, however, could be the consequence of a poorly constrained optical redshift, or a rapid outflow of gas located well clear of the jet axis, as well as the possibility that this is unassociated gas.

\subsection{Extinction effects}

So far, at least for the intermediate UV luminosity sample $\left(L_{\mathrm{UV}} \lesssim 10^{23} \mathrm{~W} \mathrm{~Hz}^{-1}\right)$, we have found no difference in the incidence of 21-cm absorption between the two AGN types, which are also indistinguishable through the absorption line profiles of the detections. In order to determine whether there is a difference in the extinction of the quasar light, in Fig. 8 we show the $V-R$ and $R-K$ colours (where available) for the published $z \geq 0.1$ searches as classified by AGN type, where, apart 
TABLE 6

The means $(\overline{\Delta v})$ and Standard deviations $(\sigma)$ For the ABSORPtion offeset from the Systemic Velocity $\left[\mathrm{km} \mathrm{s}^{-1}\right]$.

\begin{tabular}{|c|c|c|c|c|c|c|c|c|c|c|c|c|c|c|c|}
\hline & \multicolumn{3}{|c|}{ TYPE-1 } & \multicolumn{3}{|c|}{ TYPE-2 } & \multicolumn{3}{|c|}{ WHOLE SAMPLE } & \multicolumn{3}{|c|}{ Vermeulen et al. (2003) } & \multicolumn{3}{|c|}{ OTHERS } \\
\hline & $\overline{\Delta v}$ & $\sigma$ & $n$ & $\overline{\Delta v}$ & $\sigma$ & $n$ & $\overline{\Delta v}$ & $\sigma$ & $n$ & $\overline{\Delta v}$ & $\sigma$ & $n$ & $\overline{\Delta v}$ & $\sigma$ & $n$ \\
\hline$\overline{\text { Primary }}$ & -240 & 540 & 10 & -150 & 380 & 16 & -130 & 470 & 32 & -220 & 470 & 19 & 10 & 420 & 13 \\
\hline Secondary & -120 & 300 & 8 & -110 & 150 & 10 & -70 & 280 & 19 & -60 & 380 & 9 & -90 & 150 & 10 \\
\hline Both & -190 & 460 & 18 & -140 & 310 & 26 & -110 & 410 & 51 & -170 & 450 & 28 & -30 & 330 & 23 \\
\hline
\end{tabular}

TABLE 7

The statistics From Fig. 10

\begin{tabular}{lccccccc}
\hline Sample & $n$ & $\log _{10}\left(N_{\mathrm{HI}} \cdot f / T_{s}\right)$ & \multicolumn{3}{c}{$V-K$} & $S(\tau)$ \\
& & Mean & $\sigma$ & Mean & $\sigma$ & \\
\hline Whole & 58 & $<18.3$ & 0.5 & 3.9 & 1.6 & $3.63 \sigma$ \\
Detections & 26 & 18.5 & 0.6 & 4.6 & 1.6 & $2.03 \sigma$ \\
Type-1 detections & 7 & 18.5 & 0.8 & & 4.8 & 2.6 & $0.75 \sigma$ \\
$\quad$ non-detections & 19 & $<18.0$ & 0.4 & & 2.7 & 1.3 & - \\
$\quad$ exc. UV lum. & 6 & $<17.9$ & 0.3 & 3.6 & 0.7 & - \\
Type-2 detections & 15 & 18.6 & 0.6 & 4.4 & 1.0 & $1.19 \sigma$ \\
$\quad$ non-detections & 8 & $<18.2$ & 0.5 & 4.3 & 0.7 & - \\
\hline
\end{tabular}

from four type-1 outliers, we see no discernible difference

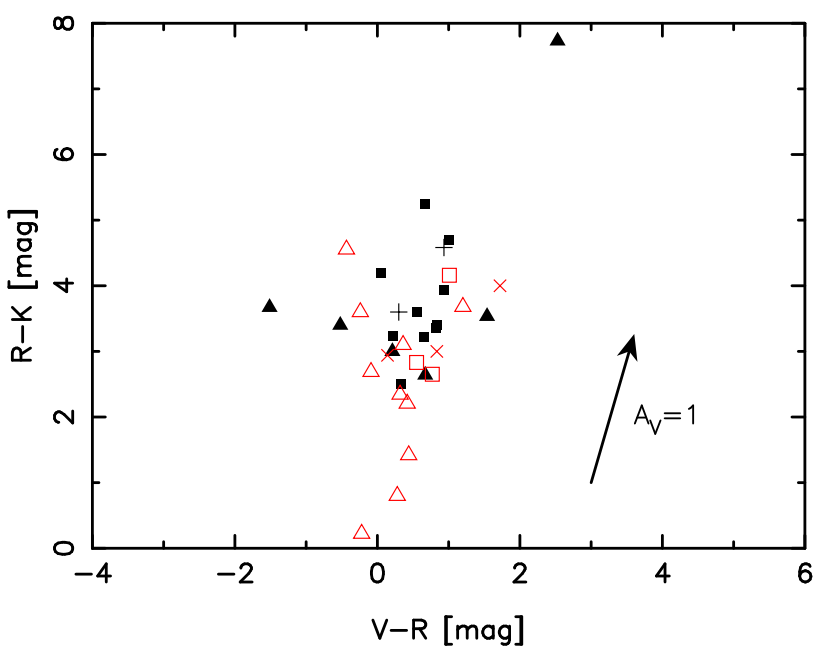

Fig. 8. - The $R-K$ colour versus the $V-R$ colour for the sample. As per Fig. 1 the filled symbols represent the 21-cm detections and the unfilled symbols the non-detections, with the triangles representing type- 1 objects and squares type-2s $(+$ and $x$ designating a non-specific AGN type for a detection and nondetection, respectively)

between the two types ${ }^{15}$. This suggests that the circumnuclear torus does not introduce a measurable degree of optical extinction, although contamination from the host galaxy starlight could lessen any apparent reddening.

In order to further examine the reddening, after correcting for the unavailable $V$ magnitudes (see Fig. 9), in Fig. 10 we show the 21-cm line strengths and limits against the optical-near-infrared colour of the source. From the statistics (Table 7), aside from the incidence of the mix of detections and non-detections (as shown

15 The outlier with the high extinction in the direction of the reddening vector is the extremely red quasar J0414+0534 (see Figs. 9 and 10. by $n)^{16}$, the only significant difference between the AGN types are the $V-K$ colours for the non-detections: Although the type- 1 detections are slightly redder than the type- $2(V-K \approx 4.8$ cf. 4.4$)$, the type- 1 non-detections could be considerably less red than those of the type2s $(V-K=2.6$, cf. 4.3). Naturally, this will be skewed by the inclusion of the $L_{\mathrm{UV}} \gtrsim 10^{23} \mathrm{~W} \mathrm{~Hz}^{-1}$ sources (Sect. 2.1.1) and omitting these raises this to $V-K=3.6$ (the "exc. UV lum." row), which, considering the $1 \sigma$ spreads, is indistinguishable from the type-2 values. Note finally, that a mean line strength of $\log _{10}\left(N_{\mathrm{HI}} \cdot f / T_{s}\right) \sim 18.5$ is exhibited for the detections of both AGN types.

On this issue, over the whole sample we find a correlation between the 21-cm line strength and the $V-K$ colour, significant at $3.63 \sigma$, Table 7$)^{17}$. This may suggest that the reddening is due to dust associated with the intervening neutral gas, rather than intrinsic to the AGN spectrum (Webster et al. 1995; Stickel et al. 1996; although see Francis et al. 2000; Whiting et al. 2001). Circumstantial evidence for this association was previously presented by Carilli et al. (1998), although we show, for the first time, a correlation between the line strength and colour. However, in light of our other findings, we know that the high UV luminous sources have low 21-cm line strengths, and these being located at the blue end of Fig. $\left[10^{18}\right.$ must drive much of the correlation. Furthermore, the overall correlation is quite fragile with the significance dropping quickly with the sample size (Table 7). This could be a reflection of the heterogeneous nature of the sample ${ }^{19}$, some of which will also be subject to contamination from starlight, although we have previously found that the molecular, rather than atomic, gas content appears to dominate the degree of reddening (Curran et al. 2006).

\section{CONCLUSIONS AND INTERPRETATION}

\subsection{Ultra-violet luminosities}

In a previous paper (Curran et al. 2008c) we found that the ultra-violet luminosity plays more of a rôle than the AGN type in the detection of 21-cm absorption in $z \geq 0.1$ radio galaxies and quasars. Specifically, to date, 21-cm absorption has never been detected in a host galaxy when $L_{\mathrm{UV}} \gtrsim 10^{23} \mathrm{~W} \mathrm{~Hz}^{-1}$. Although these high

16 The numbers give the impression that 21-cm absorption is more likely to arise in type-2 objects (cf. Fig. 1). However these are subject to the available magnitudes and many of the $K$ magnitudes are unavailable for the non-detections (Table 2).

17 The upper limits in the 21-cm line strengths (final column) are incorporated via the ASURV package.

18 All but two of the $L_{\mathrm{UV}} \lesssim 10^{23} \mathrm{~W} \mathrm{~Hz}^{-1}$ sources have $V-K<$ 3.5 .

19 And is thus not apparent in Fig. 8 

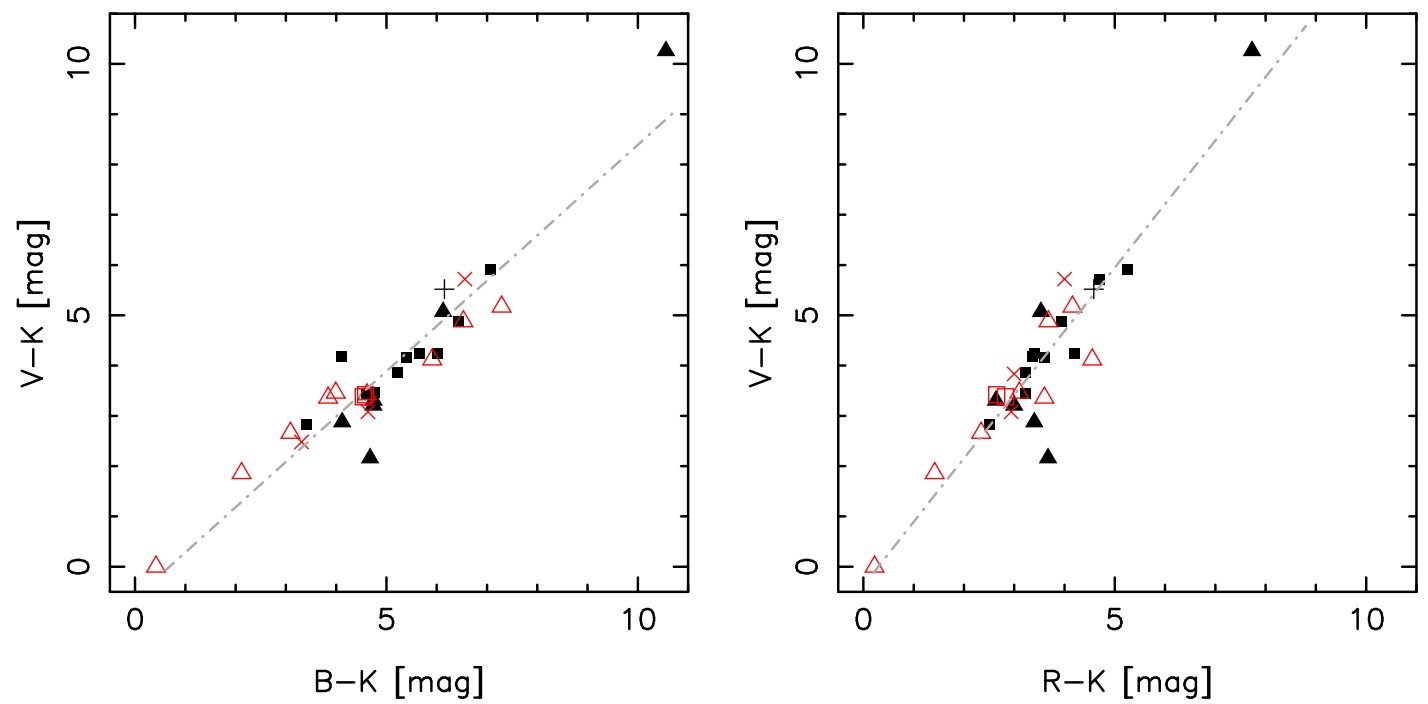

Fig. 9. - Comparison of the $V-K$ colours with the $B-K$ and $R-K$ colours for the $z_{\mathrm{em}}<3$ sample (to avoid contamination of the $B$ and $V$ bands by Lyman- $\alpha$ absorption). From the fits shown we obtain $V-K=0.90 \pm 0.01 \times(B-K)-0.62 \pm 0.29$ (significant at $5.86 \sigma$ ) and $V-K=1.26 \pm 0.02 \times(R-K)-0.37 \pm 0.29$ (significant at $5.25 \sigma$ ), which are used to convert $B-K$ and $R-K$ to $V-K$, where $V$ is unavailable. The symbols are as per Fig. 1

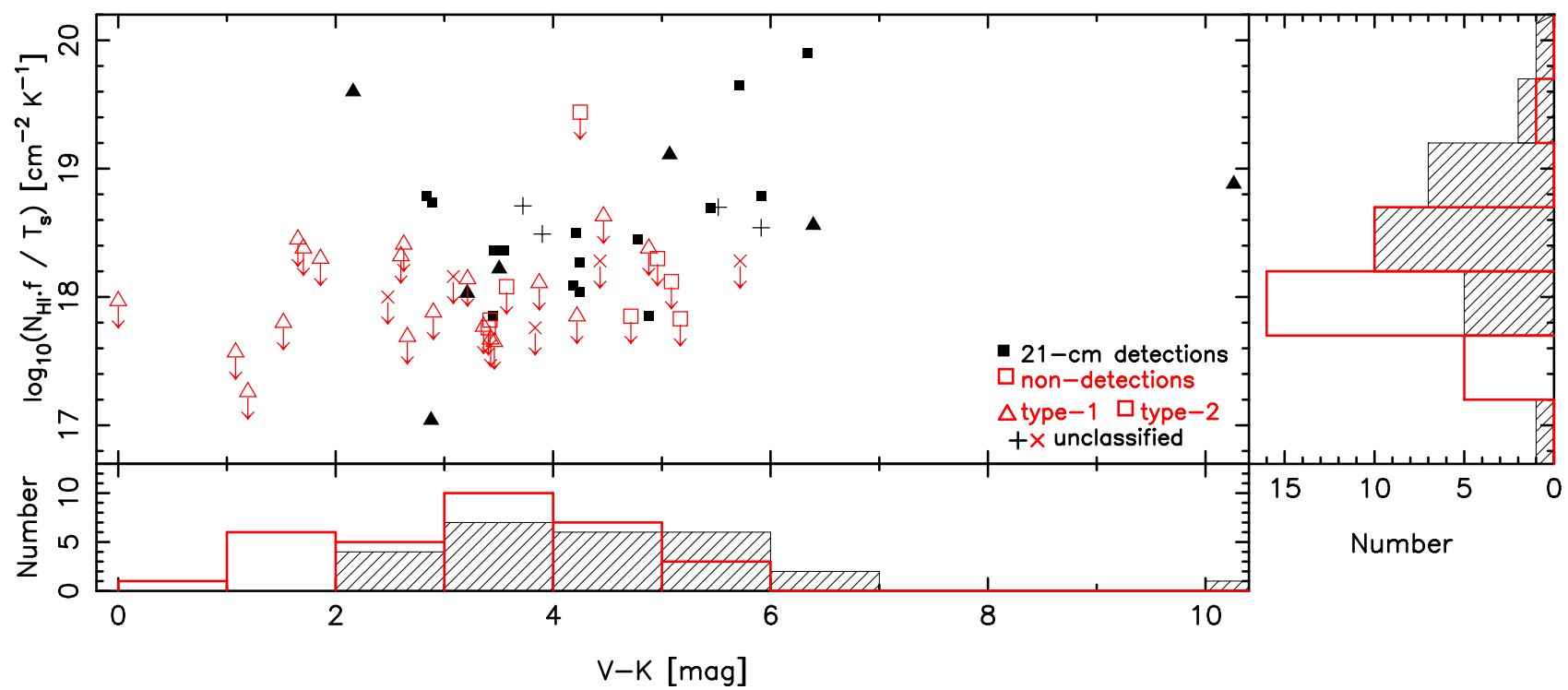

FIG. 10.- The scaled velocity integrated optical depth of the H I line $\left(1.823 \times 10^{18} \cdot \int \tau d v\right)$ versus optical-near-infrared colour for the sample. The symbols are as per Fig. [1] with the hatched histogram represent the 21-cm detections and the unfilled histogram the nondetections. Where the $V$ magnitudes are not available (Tables $6 \& 7$ of Curran et al. 2008c), we have estimated these according to the fits derived in Fig. 9 The statistics are summarised in Table 7 The outlier at $V-K=10.26$ is due to J0414+0534, which has an intervening gravitational lens, which may be responsible for some of the extreme reddening (see Curran et al. 2007 and references therein).

UV luminosities occur exclusively in type-1 objects, for the moderately UV luminous $\left(L_{\mathrm{UV}} \lesssim 10^{23} \mathrm{~W} \mathrm{~Hz}^{-1}\right.$ ) sample there is a $50 \%$ probability of detection in either AGN type, with any apparent bias against type- 1 objects being caused by the 17 high UV luminosity objects. Expanding upon these results, in this paper we show:

1. That the bias against 21-cm absorption in quasars, compared to radio galaxies, also appears to be due to UV luminosity effects: For the radio galaxies, the 21-cm detections and non-detections both arise in objects with a mean $\overline{L_{\mathrm{UV}}} \approx 2 \times 10^{20} \mathrm{~W} \mathrm{~Hz}^{-1}$, whereas the $21-\mathrm{cm}$ detected quasars have $\overline{L_{\mathrm{UV}}} \approx$ $4 \times 10^{21} \mathrm{~W} \mathrm{~Hz}^{-1}$, with the non-detected quasars having $\overline{L_{\mathrm{UV}}} \approx 5 \times 10^{22} \mathrm{~W} \mathrm{~Hz}^{-1}$.

2. Although there is this decrease in the 21-cm detection rate with increasing $L_{\mathrm{UV}}$, it is possible that the exclusive $21-\mathrm{cm}$ non-detections at $L_{\mathrm{UV}} \gtrsim 10^{23}$ $\mathrm{W} \mathrm{Hz}{ }^{-1}$ are due to the fact that highly luminous sources are believed to trace the (neutral) gas-poor elliptical galaxies. However only two of the 17 $L_{\mathrm{UV}} \gtrsim 10^{23} \mathrm{~W} \mathrm{~Hz}^{-1}$ sources are known to be located in ellipticals (although all of them could be), whereas all are known to have high ultra-violet luminosities. Therefore it is not clear whether the lack of 21-cm absorption is due to a low abundance of neutral galaxy in the host or excitation 
effects caused by the high luminosities, or indeed how these two scenarios may be related.

3. With the exclusion of the $L_{\mathrm{UV}} \gtrsim 10^{23} \mathrm{~W}$ $\mathrm{Hz}^{-1}$ sources, the skew towards the detection of 21-cm absorption in compact objects (CSO/CSS/GPS/HFP) becomes much less significant. Again, this indicates a bias introduced by the high UV luminosity sample and perhaps suggests that it is more meaningful to discuss the 21$\mathrm{cm}$ absorption incidence in terms of the rest frame ultra-violet luminosities rather than by AGN type or radio SED classifications.

\subsection{Detection rates at moderate UV luminosities}

Regarding the $L_{\mathrm{UV}} \lesssim 10^{23} \mathrm{~W} \mathrm{~Hz}^{-1}$ sources, in addition to both AGN types having a $50 \%$ detection rate, there is no evidence for the expected larger degree of reddening in the type-2 objects, which would be caused by the presence of a dusty obscuration. We do find, however, that the optical-near-infrared colour appears to be correlated with the 21-cm absorption line strength over the whole sample. These points would therefore appear to contradict the notion that the 21-cm optical depth in the hosts of high redshift galaxies and quasars is determined by the orientation of the central obscuring torus. Although, like the literature, we find a higher incidence of $21-\mathrm{cm}$ absorption in galaxies than in quasars, unlike the literature, we believe this to be a consequence of their lower ultra-violet luminosities, rather than their AGN classification ${ }^{20}$. Furthermore, the fact that the reddening is also independent of AGN type, suggests that this is also unrelated to the torus and the correlation between the reddening and the absorption line strength suggests that the bulk H I and dust share a similar (non-nuclear) distribution.

This could explain why only a fraction of $\mathrm{H}$ I absorbing AGN are detection in $\mathrm{H}_{2} \mathrm{O}$-maser emission (Tavlor et al. 2002): $\mathrm{H}_{2} \mathrm{O}$-masers are believed to arise close to the black hole in the central obscuration (Haschick et al. 1996) and thus trace type- 2 objects. Therefore, if $\mathrm{H}$ I absorption also arose in the torus, one would expect a high detection rate of $\mathrm{H}_{2} \mathrm{O}$-masers in AGN detected in $\mathrm{H}$ I absorption. However, Taylor et al. (2002) find $\mathrm{H} \mathrm{I}$ and $\mathrm{H}_{2} \mathrm{O}$ common to only 8 out of 19 objects searched and this $42 \%$ rate is very close to the overall $\mathrm{HI}$ detection rate in AGN (see Curran et al. 2008c). This therefore suggests that the lines-of-sight through the masing disk and the $\mathrm{HI}$ absorbing clouds are randomly oriented with respect to one another.

In the moderate UV luminous sample, not dominated by elliptical hosts, we therefore argue that the absorption may be occuring in the main galactic disk which must be randomly oriented with respect to the torus. In

\footnotetext{
${ }^{20}$ As discussed in Curran et al. (2008c), the exclusivity of type1 objects at $L_{\mathrm{UV}} \gtrsim 10^{23} \mathrm{~W} \mathrm{~Hz}^{-1}$ is not likely to be coincidental. They do, however, seem to be quite distinct from their lower UV
}

attempting to verify this:

1. We find no real difference in the full-width half maxima of the 21-cm absorption profiles between the two AGN types. This may suggest that these are subject to geometric effects (covering factors and radio source sizes) and thus cannot give the full kinematical picture. Although, again, absorption due to a randomly oriented disk could account for this.

2. We also find no discernible difference in the offset of the centroid of the absorption and the systemic velocity of the host between the two AGN types. Although through a sample nearly double in size, we can confirm the findings of Vermeulen et al. (2003), that on average the offsets are blue-shifted. That is, there may be evidence for outflowing gas, although, again, we see no distinction between the two AGN types.

If the galactic disk were aligned with the obscuring torus, the $50 \%$ detection rate for both AGN types suggests that absorption must occur in both galactic disks and outflows: The outflowing gas is expected to be directed along the radio jets (e.g. Begelman et al. 1984; Schulz 1988; Colbert et al. 1996, 1998), which are coincident with the axis of the torus, thus giving rise to the absorption in the type- 1 objects. The fact that we cannot discriminate between the disk and outflow absorption would suggest rapid, wide outflows of cold neutral gas, perhaps enveloping the wide ionisation cones observed in low redshift AGN (see table 1.2 of Curran 2000b 11. A $90^{\circ}$ wide outflow of cool neutral gas expanding at $\approx 200 \mathrm{~km} \mathrm{~s}^{-1}$, in which the molecular gas mass rivals that in the disk $\left(M_{\mathrm{H}} \sim 10^{9} \mathrm{M}_{\odot}\right)$, is known of in the Circinus galaxy (Curran et al. 1999), the proximate type-2 Seyfert (see also Morganti et al. 2003, 2005a for further examples).

However, it would therefore remain a mystery as to why only $50 \%$ of each AGN type (of $L_{\mathrm{UV}} \lesssim 10^{23} \mathrm{~W}$ $\mathrm{Hz}^{-1}$ ) are detected in 21-cm absorption, although the bulk absorption occuring in the galactic disk, which is randomly oriented with respect to the obscuring torus, could account for this.

This research has made use of the NASA/IPAC Extragalactic Database (NED) which is operated by the Jet Propulsion Laboratory, California Institute of Technology, under contract with the National Aeronautics and Space Administration. This research has also made use of NASA's Astrophysics Data System Bibliographic Service and ASURV Rev 1.2 (Lavalley et al. 1992), which implements the methods presented in Isobe et al. (1986).

luminosity counterparts, which exhibit the same 21-cm detection rate as the type- 2 objects.

\section{REFERENCES}

Adelman-McCarthy, J. K., Agüeros, M. A., Allam, S. S., Anderson, K. S. J., Anderson, S. F., Annis, J., Bahcall, N. A., \& MORE, B. . 2008, ApJS, 175, 297

Akujor, C. E. \& Garrington, S. T. 1995, A\&AS, 112, 235
Akujor, C. E., Spencer, R. E., Zhang, F. J., Davis, R. J., Browne, I. W. A., \& Fanti, C. 1991, MNRAS, 250, 215

Allen, D. A., Ward, M. J., \& Hyland, A. R. 1982, MNRAS, 199, 969

Antonucci, R. R. J. 1993, ARA\&A, 31, 473 
Antonucci, R. R. J. \& Miller, J. S. 1985, ApJ, 297, 621

Antonucci, R. R. J. \& Ulvestad, J. S. 1985, ApJ, 294, 158

Bahcall, J., Kirhakos, S., Saxe, D., \& Schneider, D. 1997, ApJ, 479, 642

Bahcall, J. N. \& Ekers, R. D. 1969, ApJ, 157, 1055

Baum, S. A., O'Dea, C. P., Murphy, D. W., \& de Bruyn, A. G. 1990, A\&A, 232, 19

Beasley, A. J., Gordon, D., Peck, A. B., Petrov, L., MacMillan, D. S., Fomalont, E. B., \& Ma, C. 2002, ApJS, 141, 13

Begelman, M. C., Blandford, R. D., \& Rees, M. J. 1984, Reviews of Modern Physics, 56, 255

Bellamy, M. J., Tadhunter, C. N., Morganti, R., Wills, K. A., Holt, J., Taylor, M. D., \& Watson, C. A. 2003, MNRAS, 344, L80

Bergman, P., Aalto, S., Black, J. H., \& Rydbeck, G. 1992, A\&A, 265, 403

Bondi, M., Garrett, M. A., \& Gurvits, L. I. 1998, MNRAS, 297, 559

Bondi, M., Padrielli, L., Fanti, R., Ficarra, A., Gregorini, L., Mantovani, F., Bartel, N., Romney, J. D., Nicolson, G. D., \& Weiler, K. W. 1996, A\&A, 308, 415

Browne, I. W. A., Orr, M. J. L., Davis, R. J., Foley, A., Muxlow, T. W. B., \& Thomasson, P. 1982, MNRAS, 198, 673

Buchanan, C. L., McGregor, P. J., Bicknell, G. V., \& Dopita, M. A. 2006, AJ, 132, 27

Carballo, R., Sánchez, S. F., González-Serrano, J. I., Benn, S. R., \& Vigotti, M. 1998, ApJ, 115, 1234

Carilli, C. L., Menten, K. M., Reid, M. J., Rupen, M. P., \& Yun, M. S. 1998, ApJ, 494, 175

Carilli, C. L., Perlman, E. S., \& Stocke, J. T. 1992, ApJ, 400, L13

Carilli, C. L., Wang, R., Hoven, M., Dwarakanath, K., Chengalur, J., \& Wyithe, S. 2007, AJ, 133, 2841

Colbert, E. J. M., Baum, S. A., Gallimore, J. F., O'Dea, C. P., \& Christensen, J. A. 1996, ApJ, 467, 551

Colbert, E. J. M., Baum, S. A., O'Dea, C. P., \& Veilleux, S. 1998, ApJ, 496, 786

Conway, J. 1997, in Gigahertz Peaked Spectrum and Compact Steep Spectrum Radio Sources, ed. I. A. G. Snellen, R. T Schilizzi, H. J. A. Roettgering, \& M. N. Bremer, 198-207

Conway, J. E. \& Blanco, P. R. 1995, ApJ, 449, L131

Curran, S. J. 2000a, A\&AS, 144, 271

-. 2000b, PhD thesis, Chalmers University of Technology

Curran, S. J., Darling, J. K., Bolatto, A. D., Whiting, M. T., Bignell, C., \& Webb, J. K. 2007, MNRAS, 382, L11

Curran, S. J., Johansson, L. E. B., Rydbeck, G., \& Booth, R. S. 1998, A\&A, 338, 863

Curran, S. J., Koribalski, B. S., \& Bains, I. 2008a, MNRAS, 389, 63

Curran, S. J., Rydbeck, G., Johansson, L. E. B., \& Booth, R. S. 1999, A\&A, 344, 767

Curran, S. J., Tzanavaris, P., Darling, J. K., Whiting, M. T., Webb, J. K., Bignell, C., Athreya, R., \& Murphy, M. T. 2010, MNRAS, 402, 35

Curran, S. J. \& Webb, J. K. 2006, MNRAS, 371, 356

Curran, S. J., Whiting, M., Murphy, M. T., Webb, J. K., Longmore, S. N., Pihlström, Y. M., Athreya, R., \& Blake, C. 2006, MNRAS, 371,431

Curran, S. J., Whiting, M. T., \& Webb, J. K. 2008b, in The Central Kiloparsec, Active Galactic Nuclei and Their Hosts (Memorie della Societa Astronomica Italiana, Vol. 79), 1113

Curran, S. J., Whiting, M. T., Wiklind, T., Webb, J. K., Murphy, M. T., \& Purcell, C. R. 2008c, MNRAS, 391, 765

Dallacasa, D. 2003, PASA, 20, 79

Dallacasa, D., Fanti, C., Fanti, R., Schilizzi, R. T., \& Spencer, R. E. 1995, A\&A, 295, 27

de Waard, G. J., Strom, R. G., \& Miley, G. K. 1985, A\&A, 145, 479

di Serego Alighieri, S., Gavazzi, G., Giovanardi, C., Giovanelli, R., Grossi, M., Haynes, M. P., Kent, B. R., Koopmann, R. A., Pellegrini, S., Scodeggio, M., \& Trinchieri, G. 2007, A\&A, 474, 851

Drinkwater, M. J., Webster, R. L., Francis, P. J., Condon, J. J., Ellison, S. L., Jauncey, D. L., Lovell, J., Peterson, B. A., \& Savage, A. 1997, MNRAS, 284, 85

Dunlop, J. S., McLure, R. J., Kukula, M. J., Baum, S. A., O'Dea, C. P., \& Hughes, D. H. 2003, 340, 1095

Emonts, B., Morganti, R., Oosterloo, T., Holt, J., Tadhunter, C., van der Hulst, J., Ojha, R., \& Sadler, E. 2008, MNRAS, 387, 197
Fanti, C., Fanti, R., Dallacasa, D., Schilizzi, R. T., Spencer, R. E., \& Stanghellini, C. 1995, A\&A, 302, 317

Fanti, C., Fanti, R., Parma, P., Venturi, T., Schilizzi, R. T., Nan Rendong, Spencer, R. E., Muxlow, T. W. B., \& van Breugel, W. 1989, A\&A, 217, 44

Fanti, R., Fanti, C., Schilizzi, R. T., Spencer, R. E., Nan Rendong, Parma, P., van Breugel, W. J. M., \& Venturi, T. 1990, A\&A, 231,333

Fey, A. L., Clegg, A. W., \& Fomalont, E. B. 1996, ApJS, 105, 299 Field, G. B. 1959, ApJ, 129, 536

Floyd, D. J. E., Kukula, M. J., Dunlop, J. S., McLure, R. J., Miller, L., Percival, W. J., Baum, S. A., \& O'Dea, C. P. 2004, MNRAS, 355,196

Fomalont, E. B., Frey, S., Paragi, Z., Gurvits, L. I., Scott, W. K., Taylor, A. R., Edwards, P. G., \& Hirabayashi, H. 2000, ApJS, 131,95

Fomalont, E. B., Petrov, L., MacMillan, D. S., Gordon, D., \& Ma, C. 2003, AJ, 126, 2562

Francis, P. J., Whiting, M. T., \& Webster, R. L. 2000, PASA, 17, 56

Gallimore, J. F., Baum, S. A., O'Dea, C. P., Pedlar, A., \& Brinks, E. 1999, ApJ, 524, 684

Giovannini, G., Feretti, L., Gregorini, L., \& Parma, P. 1988, A\&A, 199, 73

Greenhill, L. J., Booth, R. S., Ellingsen, S. P., Herrnstein, J. R., Jauncey, D. L., McCulloch, P. M., Moran, J. M., Norris, R. P., Reynolds, J. E., \& Tzioumis, A. K. 2003, ApJ, 590, 162

Grossi, M., di Serego Alighieri, S., Giovanardi, C., Gavazzi, G., Giovanelli, R., Haynes, M. P., Kent, B. R., Pellegrini, S., Stierwalt, S., \& Trinchieri, G. 2009, A\&A, 498, 407

Gupta, N. \& Saikia, D. J. 2006a, MNRAS, 370, L80

-. 2006b, MNRAS, 370, 738

Gupta, N., Salter, C. J., Saikia, D. J., Ghosh, T., \& Jeyakumar, S. 2006, MNRAS, 373, 972

Haschick, A. D., Baan, W. A., \& Peng, E. W. 1996, ApJ, 437, L35

Hutchings, J. B., Crampton, D., Morris, S. L., Durand, D., \& Steinbring, E. 1999, AJ, 117, 1109

Irwin, J. A. \& Sofue, Y. 1992, ApJ, 396, L75

Ishwara-Chandra, C. H., Dwarakanath, K. S., \& Anantharamaiah, K. R. 2003, JA\&A, 24, 37

Isobe, T., Feigelson, E., \& Nelson, P. 1986, ApJ, 306, 490

Israel, F. P., Van Der Werf, P. P., Hawarden, T. G., \& Aspin, C. 1998, A\&A, 336, 433

Jackson, C. A., Wall, J. V., Shaver, P. A., Kellermann, K. I., Hook, I. M., \& Hawkins, M. R. S. 2002, A\&A, 386, 97

Jaffe, W. \& McNamara, B. R. 1994, ApJ, 434, 110

Jenkins, C. J., Pooley, G. G., \& Riley, J. M. 1977, Mem. R. Astr Soc., 84, 61

Kanekar, N. \& Chengalur, J. N. 2003, A\&A, 399, 857

Kanekar, N., Lane, W. M., Momjian, E., Briggs, F. H., \& Chengalur, J. N. 2009, MNRAS, 394, L61

Keel, W. C. 1980, AJ, 85, 198

Kohno, K., Kawabe, R., Tosaki, T., \& Okumura, S. K. 1996, ApJ, 461, L29

Kotilainen, J. K. \& Falomo, R. 2000, A\&A, 364, 70

Kukula, M. J., Dunlop, J. S., McLure, R. J., Miller, L., Percival, W. J., Baum, S. A., \& O'Dea, C. P. 2001, MNRAS, 326, 1533

Lane, W. M., Briggs, F. H., \& Smette, A. 2000, ApJ, 532, 146

Lavalley, M. P., Isobe, T., \& Feigelson, E. D. 1992, in BAAS, Vol. 24, 839-840

Lehnert, M. D., Miley, G. K., Sparks, W. B., Baum, S. A., Biretta, J., Golombek, D., de Koff, S., Macchetto, F. D., \& McCarthy, P. J. 1999, ApJS, 123, 351

Lipovetsky, V. A., Neizvestny, S. I., \& Neizvestnaya, O. M. 1988, Soobshcheniya Spetsial'noj Astrofizicheskoj Observatorii, 55, 5

Lister, M. L., Kellermann, K. I., Vermeulen, R. C., Cohen, M. H., Zensus, J. A., \& Ros, E. 2003, ApJ, 584, 135

Lüdke, E., Garrington, S. T., Spencer, R. E., Akujor, C. E., Muxlow, T. W. B., Sanghera, H. S., \& Fanti, C. 1998, MNRAS, 299,467

Miller, J. S. 1981, PASP, 93, 681

Miller, J. S. \& Goodrich, B. F. 1987, BAAS, 19, 695

Mirabel, I. F. 1989, ApJ, 340, L13

Moore, C. B., Carilli, C. L., \& Menten, K. M. 1999, ApJ, 510, L87

Morganti, R., de Zeeuw, P. T., Oosterloo, T. A., McDermid, R. M., Krajnović, D., Cappellari, M., Kenn, F., Weijmans, A., \& Sarzi, M. 2006, MNRAS, 371, 157 
Morganti, R., Holt, J., Saripalli, L., Oosterloo, T. A., \& Tadhunter, C. N. 2007, A\&A, 476, 735

Morganti, R., Oosterloo, T. A., Emonts, B. H. C., van der Hulst, J. M., \& Tadhunter, C. N. 2003, ApJ, 593, L69

Morganti, R., Oosterloo, T. A., Tadhunter, C. N., van Moorsel, G., \& Emonts, B. 2005a, A\&A, 439, 521

Morganti, R., Oosterloo, T. A., Tadhunter, C. N., van Moorsel, G., Killeen, N., \& Wills, K. A. 2001, MNRAS, 323, 331

Morganti, R., Tadhunter, C. N., \& Oosterloo, T. A. 2005b, A\&A, 444, L9

Mundell, C. G., Wrobel, J. M., Pedlar, A., \& Gallimore, J. F. 2003, ApJ, 583, 192

Murphy, D. W., Browne, I. W. A., \& Perley, R. A. 1993, MNRAS, 264,298

Mutel, R. L., Phillips, R. B., \& Skuppin, R. 1981, AJ, 86, 1600

Myers, S. T. \& Scoville, N. Z. 1987, ApJ, 312, L39

Nagar, N. M. \& Wilson, A. S. 1999, ApJ, 516, 97

Neff, S. G., Roberts, L., \& Hutchings, J. B. 1995, ApJS, 99, 349

Nyland, K., Young, L., \& Lucero, D. 2009, in Bulletin of the American Astronomical Society, Vol. 41, 232

O'Dea, C. P. 1998, PASP, 110, 493

O'Dea, C. P. \& Baum, S. A. 1997, AJ, 113, 148

Oosterloo, T. A., Morganti, R., Sadler, E. M., van der Hulst, T., \& Serra, P. 2007, A\&A, 465, 787

Oosterloo, T. A., Morganti, R., Sadler, E. M., Vergani, D., \& Caldwell, N. 2002, AJ, 123, 729

Orienti, M., Morganti, R., \& Dallacasa, D. 2006, A\&A, 457, 531

Osterbrock, D. E. 1978, Proc. Nat. Acad. Sci., 75, 540

Owsianik, I., Conway, J. E., \& Polatidis, A. G. 1998, A\&A, 336, L37

Pearson, T. J. \& Readhead, A. C. S. 1988, ApJ, 328, 114

Peck, A. B., Taylor, G. B., \& Conway, J. E. 1999, ApJ, 521, 103

Peck, A. B., Taylor, G. B., Fassnacht, C. D., Readhead, A. C. S., \& Vermeulen, R. C. 2000, ApJ, 534, 104

Peng, C. Y., Impey, C. D., Rix, H.-W., Falco, E. E., Keeton, C. R., Kochanek, C. S., Lehár, J., \& McLeod, B. A. 2006, New Astronomy Reviews, 50, 689

Perlman, E. S., Carilli, C. L., Stocke, J. T., \& Conway, J. 1996, AJ, 111, 1839

Petrov, L., Kovalev, Y. Y., Fomalont, E., \& Gordon, D. 2005, AJ, 129,1163

Phillips, R. B. \& Mutel, R. L. 1981, ApJ, 244, 19

Pihlström, Y. M., Conway, J. E., \& Vermeulen, R. C. 2003, A\&A, 404, 871

Pihlström, Y. M., Baan, W. A., Darling, J., \& Klöckner, H.-R. 2005, ApJ, 618, 705

Plante, R. L., Lo, K. Y., Roy, J.-R., Martin, P., \& Noreau, L. 1991, ApJ, 381, 110

Polatidis, A. G., Wilkinson, P. N., Xu, W., Readhead, A. C. S., Pearson, T. J., Taylor, G. B., \& Vermeulen, R. C. 1995, ApJS, 98,1

Reid, R. I., Kronberg, P. P., \& Perley, R. A. 1999, ApJS, 124, 285

Ridgway, S. E., Heckman, T. M., Calzetti, D., \& Lehnert, M. 2001, ApJ, 550, 122

Sadler, E. M., Oosterloo, T., \& Morganti, R. 2002, in Astronomical Society of the Pacific Conference Series, Vol. 273, The Dynamics, Structure \& History of Galaxies: A Workshop in Honour of Professor Ken Freeman, ed. G. S. Da Costa \& H. Jerjen, 215

Saikia, D. J., Jeyakumar, S., Salter, C. J., Thomasson, P., Spencer, R. E., \& Mantovani, F. 2001, MNRAS, 321, 37

Saikia, D. J., Jeyakumar, S., Wiita, P. J., Sanghera, H. S., \& Spencer, R. E. 1995, MNRAS, 276, 1215

Saikia, D. J. \& Kulkarni, A. R. 1998, MNRAS, 298, L45

Sanghera, H. S., Saikia, D. J., Luedke, E., Spencer, R. E., Foulsham, P. A., Akujor, C. E., \& Tzioumis, A. K. 1995, A\&A, 295,629

Schmitt, H. R., Kinney, A. L., Storchi-Bergmann, T., \& Antonucci, R. 1997, ApJ, 477, 623

Schulz, H. 1988, A\&A, 203, 233
Skrutskie, M. F., Cutri, R. M., Stiening, R., Weinberg, M. D., Schneider, S., Carpenter, J. M., Beichman, C., \& Capps, R. . M. 2006, AJ, 131, 1163

Smith, E. P. \& Heckman, T. M. 1989, ApJS, 69, 365

Spencer, R. E., McDowell, J. C., Charlesworth, M., Fanti, C., Parma, P., \& Peacock, J. A. 1989, MNRAS, 240, 657

Stanghellini, C., Dallacasa, D., O'Dea, C. P., Baum, S. A., Fanti, R., \& Fanti, C. 2001, A\&A, 377, 377

Stanghellini, C., O'Dea, C. P., \& Murphy, D. W. 1999, A\&AS, 134, 309

Stickel, M., Rieke, G. H., Kühr, H., \& Rieke, M. J. 1996, ApJ, 468, 556

Strom, R. G., Riley, J. M., Spinrad, H., van Breugel, W. J. M., Djorgovski, S., Liebert, J., \& McCarthy, P. J. 1990, A\&A, 227, 19

Tacconi, L. J., Genzel, R., Blietz, M., Cameron, M., Harris, A. I., \& Madden, S. 1994, ApJ, 426, L77

Tadhunter, C., Dickson, R., Morganti, R., Robinson, T. G., Wills, K., Villar-Martin, M., \& Hughes, M. 2002, MNRAS, 330, 977

Taylor, G. B., Marr, J. M., Pearson, T. J., \& Readhead, A. C. S. 2000, ApJ, 541, 112

Taylor, G. B., Peck, A. B., Henkel, C., Falcke, H., Mundell, C. G., O'Dea, C. P., Baum, S. A., \& Gallimore, J. F. 2002, ApJ, 574, 88

Taylor, G. B., Readhead, A. C. S., \& Pearson, T. J. 1996a, ApJ, 463, 95

Taylor, G. B. \& Vermeulen, R. C. 1997, 485, L9

Taylor, G. B., Vermeulen, R. C., Pearson, T. J., Readhead, A. C. S., Henstock, D. R., Browne, I. W. A., \& Wilkinson, P. N. 1994, ApJS, 95, 345

Taylor, G. L., Dunlop, J. S., Hughes, D. H., \& Robson, E. I. 1996b, MNRAS, 283, 930

Thomasson, P., Saikia, D. J., \& Muxlow, T. W. B. 2003, MNRAS, 341,91

Tzioumis, A., King, E., Morganti, R., Dallacasa, D., Tadhunter, C., Fanti, C., Reynolds, J., Jauncey, D., Preston, R., McCulloch, P., Tingay, S., Edwards, P., Costa, M., Jones, D., Lovell, J., Clay, R., Meier, D., Murphy, D., Gough, R., Ferris, R., White, G., \& Jones, P. 2002, A\&A, 392, 841

Ulvestad, J. S., Johnston, K. J., \& Weiler, K. W. 1983, ApJ, 266, 18

Urry, C. M. \& Padovani, P. 1995, PASP, 107, 803

Urry, C. M., Scarpa, R., O'Dowd, M., Falomo, R., Pesce, J. E., \& Treves, A. 2000, ApJ, 532, 816

Uson, J. M., Bagri, D. S., \& Cornwell, T. J. 1991, PhRvL, 67, 3328

van Breugel, W. J. M., Fanti, C., Fanti, R., Stanghellini, C., Schilizzi, R. T., \& Spencer, R. E. 1992, A\&A, 256, 56

van Gorkom, J. H., Knapp, G. R., Ekers, R. D., Ekers, D. D., Laing, R. A., \& Polk, K. S. 1989, AJ, 97, 708

Vermeulen, R. C., Pihlström, Y. M., Tschager, W., de Vries, W. H., Conway, J. E., Barthel, P. D., Baum, S. A., Braun, R., Bremer, M. N., Miley, G. K., O'Dea, C. P., Roettgering, H. J. A., Schilizzi, R. T., Snellen, I. A. G., \& Taylor, G. B. 2003, A\&A, 404, 861

Wall, J. V. \& Peacock, J. A. 1985, MNRAS, 216, 173

Webster, R. L., Francis, P. J., Peterson, B. A., Drinkwater, M. J., \& Masci, F. J. 1995, Nat, 375, 469

White, R. L., Becker, R. H., Helfand, D. J., \& Gregg, M. D. 1997, ApJ, 475, 479

Whiting, M. T., Webster, R. L., \& Francis, P. J. 2001, MNRAS, 323,718

Whyborn, N. D., Browne, I. W. A., Wilkinson, P. N., Porcas, R. W., \& Spinrad, H. 1985, MNRAS, 214, 55

Xu, W., Readhead, A. C. S., Pearson, T. J., Polatidis, A. G., \& Wilkinson, P. N. 1995, ApJS, 99, 297

Young, J. S. \& Scoville, N. 1991, ARA\&A, 29, 581

Zensus, J. A., Ros, E., Kellermann, K. I., Cohen, M. H., Vermeulen, R. C., \& Kadler, M. 2002, AJ, 124, 662 